PACS: $67.40 . \mathrm{Bz}, 67.40 . \mathrm{Pm}$

\title{
A CONVECTIVE MODEL OF A ROTON
}

\author{
V.I. Tkachenko ${ }^{1,2}$ \\ ${ }^{1)}$ National Science Center "Kharkov Institute of Physics and Technology" \\ The National Academy of Sciences of Ukraine \\ 61108, Kharkov 1, Akademicheskaya str., tel./fax 8-057-349-10-78 \\ ${ }^{2)}$ V.N. Karazin Kharkiv National University, \\ 61022, Kharkov,4, Svobody sq., tel./fax 8-057-705-14-05 \\ E-mail: tkachenko@kipt.kharkov.ua \\ Received February 17, 2017
}

A convective model describing the nature and structure of the roton is proposed. According to the model, the roton is a cylindrical convective cell with free horizontal boundaries. On the basis of the model, the characteristic geometric dimensions of the roton are estimated, and the spatial distribution of the velocity of the helium atoms and the perturbed temperature inside are described. It is assumed that the spatial distribution of rotons has a horizontally multilayer periodic structure, from which follows the quantization of the energy spectrum of rotons. The noted quantization allows us to adequately describe the energy spectrum of rotons. The convective model is quantitatively confirmed by experimental data on the measurement of the density of the normal component of helium II, the scattering of neutrons and light by helium II. The use of a convective model for describing the scattering of light by helium II made it possible to estimate the dipole moment of the roton, as well as the number of helium atoms participating in the formation of the roton.

KEY WORDS: superfluid helium, convection, elementary convective cell, roton, energy spectrum of helium II, density of the normal component of helium II, neutron scattering, light scattering, dipole momentum

\author{
КОНВЕКТИВНА МОДЕЛЬ РОТОНА \\ B.I. Ткаченко ${ }^{1,2}$ \\ 1) Національний науковий центр "Харківський фізико-технічний інститут" \\ Національної академї̈ наук України \\ 61108, м. Харків, вул. Академічна 1, tel./fax 8-057-349-10-78 \\ 2) Харківський національний університет імені В.Н. Каразіна \\ 61022, м. Харків, пл. Свободи, 4, tel./fax 8-057-705-14-05
}

Запропоновано конвективну модель, що описує природу і структуру ротона. Згідно моделі ротон є циліндрична конвективна комірка з вільними горизонтальними межами. На підставі моделі оцінені характерні геометричні розміри ротона та описано просторовий розподіл швидкості атомів гелію та збуреної температури всередині нього. Передбачається, що просторовий розподіл ротонів має горизонтально-багатошарову періодичну структуру, $з$ якої випливає квантування енергетичного спектру ротонів. Це квантування дозволяє адекватно описати енергетичний спектр ротонів. Конвективна модель кількісно підтверджується експериментальними даними для вимірювання щільності нормальної компоненти гелію II, розсіюванню нейтронів і світла на гелії ІІ. Використання конвективної моделі для опису розсіяння світла на гелії II дозволило оцінити дипольний момент ротона, а також кількість атомів гелію, що беруть участь у формуванні ротона.

КЛЮЧОВІ СЛОВА: надплинний гелій, конвекція, елементарний конвективний осередок, ротон, енергетичний спектр гелію II, щільність нормальної компоненти гелію II, розсіювання нейтронів, розсіювання світла, дипольний момент

\author{
КОНВЕКТИВНАЯ МОДЕЛЬ РОТОНА \\ В.И. Ткаченко ${ }^{1,2}$ \\ 1) Национальный научный центрр "Харьковский физико-технический институт" \\ Национальной академии наук Украины \\ 61108, г. Харьков, ул. Академическая 1, tel./fax 8-057-349-10-78 \\ 2) Харьковский национальный университет имени В.Н. Каразина \\ 61022, г. Харьков, пл. Свободы 4, tel./fax 8-057-705-14-05
}

Предложена конвективная модель, описывающая природу и структуру ротона. Согласно модели ротон является цилиндрической конвективной ячейкой со свободными горизонтальными границами. На основании модели оценены характерные геометрические размеры ротона, и описано пространственное распределение скорости атомов гелия и возмущенной температуры внутри него. Предполагается, что пространственное распределение ротонов имеет горизонтально-многослойную периодическую структуру, из которой следует квантование энергетического спектра ротонов. Отмеченное квантование позволяет адекватно описать энергетический спектр ротонов. Конвективная модель количественно подтверждается экспериментальными данными по измерению плотности нормальной компоненты гелия II, рассеянию нейтронов и света на гелии II. Использование конвективной модели для описания рассеяния света на гелии II позволило оценить дипольный момент ротона, а также количество участвующих в формировании ротона атомов гелия.

КЛЮЧЕВЫЕ СЛОВА: сверхтекучий гелий, конвекция, элементарная конвективная ячейка, ротон, энергетический спектр гелия II, плотность нормальной компоненты гелия II, рассеяние нейтронов, рассеяние света, дипольный момент 


\section{ЭКСПЕРИМЕНТЫ ПО ОПРЕДЕЛЕНИЮ ЭНЕРГЕТИЧЕСКОГО СПЕКТРА РОТОНОВ}

В работе [1] Ландау представил количественную теорию сверхтекучего гелия, которая описала практически все известные к моменту ее появления экспериментальные результаты, и предсказала ряд новых явлений.

Согласно этой теории, при температуре ниже $\lambda$ - точки $\left(T_{\lambda}=2,17 K\right)$, гелий, который принято называть гелий II, одновременно содержит в себе два вида тепловых возбуждений: фононы и ротоны.

Первый вид тепловых возбуждений (фононы) существует в длинноволновой части спектра и характеризуется линейным законом зависимости энергии такой квазичастицы от ее импульса: $\varepsilon(p)=c p$, где $c$ - скорость обычного звука в гелии II, равная приблизительно $240 \mathrm{M} / c е \kappa, \varepsilon(p)$ и $p$ - энергия и импульс квазичастицы соответственно.

Второй вид тепловых возбуждений (ротоны) существует в более коротковолновой относительно фононов части спектра. При этом постулируется, что связь между энергией $\varepsilon(p)$ и импульсом $p$ в этой части энергетического спектра характеризуется параболической зависимостью: $\varepsilon(p)=\Delta+\left(p-p_{0}\right)^{2} / 2 \mu, \quad$ где постоянные определяются следующим образом: $\Delta$ - ширина энергетической щели между невозбужденным и возбужденным уровнями, $p_{0}$ - импульс ротона, $\mu$ - эффективная масса ротона (в единицах массы атома ${ }^{4} H e$ : $m_{H e}=6.646 \cdot 10^{-24}$ г), на котором рассеиваются нейтроны.

Постулируемая зависимость энергии от импульса в коротковолновой части спектра была подтверждена, по мнению авторов ряда публикаций (см., например, [2-4]), в экспериментах с помощью нейтронографического анализа, когда зондирующий пучок монохроматических нейтронов с определенной длиной волны рассеивался на гелии II. В этих экспериментах были определены значения постоянных энергетического спектра Ландау, которые сведены в таблицу 1:

Таблица 1.

Значения постоянных энергетического спектра ротонов

\begin{tabular}{|l|l|l|l|}
\hline$\Delta / k=9,6, K$ & $p_{0} / \hbar=1,95, \AA^{-1}$ & $\mu=1,06 m_{H e}$ & {$[2]$} \\
\hline$\Delta / k=8,1 \pm 0,4, K$ & $p_{0} / \hbar=1,9 \pm 0,03, \AA^{-1}$ & $\mu=0,16 \pm 0,02 m_{H e}$ & {$[3]$} \\
\hline$\Delta / k=8,65 \pm 0,04, K$ & $p_{0} / \hbar=1,92 \pm 0,01, \AA^{-1}$ & $\mu=0,16 \pm 0,01 m_{H e}$ & {$[4]$} \\
\hline
\end{tabular}

где $m_{H e}=6.646483612210^{-24}$ г - масса атома гелия [5], $k$ - постоянная Больцмана.

Согласно [4] ширина энергетической щели $\Delta / k$ не является постоянной величиной, а уменьшается с ростом температуры по закону $\Delta / k=8,68-0,0084 \cdot T^{7} K$.

Как следует из таблицы 1 , значения констант энергетического спектра имеют разные значения.

Это несоответствие указывает на необходимость придания физического смысла постулируемой коротковолновой части энергетического спектра.

\section{МОДЕЛИ СТРОЕНИЯ РОТОНА}

Набор большой экспериментальной базы данных по теплофизическим параметрам ротонов до настоящего времени не завершился созданием его физической модели.

На протяжении всего периода исследования гелия II различными методами не прекращаются попытки описать структуру и свойства ротона.

Так, например, Р. Фейнман предложил модель ротона в виде вихревого кольца, состоящего из шести атомов ${ }^{4} \mathrm{He}$, и расположенных так вдоль линии кольца, что промежутки между ними порядка диаметра атома гелия. Каждый находящийся в кольце атом синхронно вращается, располагаясь то в первоначальном положении, то занимая соседний, например, левый промежуток. Характерный размер вихревого кольца порядка среднего атомного расстояния в жидком гелии II [6].

Другая модель ротонной структуры [7] основана на предположении о том, что в гелии II при температуре $0,6 \leq T(K) \leq 1,2$ существуют стабильные кластеры, которые являются связанными состояниями некоторого числа $N_{c}$ атомов ${ }^{4} \mathrm{He}\left(N_{c}>>1\right)$. Число $N_{c}$ атомов в таком ротонном кластере может быть определено по минимуму свободной энергии, которая представляет собой условие устойчивости ротона. По данным [7] стабильный кластер должен иметь близкую к сферической форму, и число атомов гелия - $N_{c}=13$. Средний радиус такого сферического кластера оценивается величиной 5,22 А.

Однако, в описанных моделях ротона (вихревая и сферическая) отсутствует какое-либо описания физической природы сил, удерживающих атомы гелия в кольце или в сфере. 


\section{СВОБОДНАЯ ЭНЕРГИЯ, ЭНЕРГИЯ, ЧИСЛО ФОНОНОВ И ФОНОННАЯ ЧАСТЬ ПЛОТНОСТИ} НОРМАЛЬНОЙ КОМПОНЕНТЫ ГЕЛИЯ ІІ

Свободная энергия Бозе - газа в единице объема определяется выражением $[1,8,9]$ :

$$
F(T)=-\frac{k T}{(2 \pi \hbar)^{3}} \int_{0}^{\infty} \int_{0}^{\pi} \int_{-\pi}^{\pi} \ln (1+n(\varepsilon)) d \vec{p}
$$

где $n(\varepsilon(p))=\left(e^{\frac{\varepsilon(p)}{k T}}-1\right)^{-1}$ - функция распределения Планка для частиц с энергией $\varepsilon(p), d \vec{p}=p^{2} d p d o$ элемент объема в пространстве импульсов $\vec{p}, d o$ - элемент телесного угла.

Для фононов $\varepsilon(p)=c p$, где $c$ - скорость звука в гелии II, $p$ - модуль импульса фонона.

После интегрирования по частям в (1), для свободной энергии фононов имеем:

$$
\begin{aligned}
& F_{p h}(T)=-\frac{k T}{(2 \pi \hbar)^{3}} \int_{0}^{\infty} \int_{0}^{\pi} \int_{-\pi}^{\pi} \ln (1+n(c p)) d \vec{p}=-\frac{k T}{(2 \pi \hbar)^{3}}\left[\left.\int_{0}^{\pi} \int_{-\pi}^{\pi} \ln (1+n(c p)) \frac{p^{3}}{3}\right|_{0} ^{\infty} d o+\right. \\
& \left.+\frac{1}{3 k T} \int_{0}^{\infty} \int_{0}^{\pi} \int_{-\pi}^{\pi} n p \frac{d \varepsilon(p)}{d p} d \vec{p}\right]=-\frac{1}{3(2 \pi \hbar)^{3}} \int_{0}^{\infty} \int_{0}^{\pi} \int_{-\pi}^{\pi} n p c d \vec{p}
\end{aligned}
$$

Первое слагаемое в квадратных скобках на верхнем и нижнем пределах равно нулю.

Интегрирование (2) дает выражение:

$$
\begin{aligned}
& F_{p h}(T)=-\frac{c}{3(2 \pi \hbar)^{3}} \int_{0}^{\infty} \int_{0}^{\pi} \int_{-\pi}^{\pi}\left(e^{\frac{c p}{k T}}-1\right)^{-1} p^{3} d p \sin (\vartheta) d \vartheta d \varphi=-\frac{4 \pi}{3(2 \pi \hbar)^{3}} k T\left(\frac{k T}{c}\right)^{3} \int_{0}^{\infty} \frac{x^{3}}{e^{x}-1} d x= \\
& =-\frac{4 \pi^{5}}{45(2 \pi \hbar)^{3}} k T\left(\frac{k T}{c}\right)^{3}=-\frac{E_{p h}}{3}
\end{aligned}
$$

где $E_{p h}=\frac{4 \pi^{5}}{15} k T\left(\frac{k T}{2 \pi \hbar c}\right)^{3}$ - энергия фонов в единичном объеме гелия II.

При этом, число фононов в единичном объеме определяется выражением:

$$
N_{p h}(T)=\frac{1}{(2 \pi \hbar)^{3}} \int_{0}^{\infty} \int_{0}^{\pi} \int_{0}^{2 \pi}\left(e^{\frac{c p}{k T}}-1\right)^{-1} p^{2} d p \sin (\vartheta) d \vartheta d \varphi=2 \zeta(3) 4 \pi\left(\frac{k T}{2 \pi \hbar c}\right)^{3}
$$

где $\zeta(x)$ - дзета-функция Римана от аргумента $x, \zeta(3) \approx 2.4041$.

Таким образом, энергия фононов связана с числом фононов в единичном объеме гелия II выражением:

$$
E_{p h}=\frac{\pi^{4} k T}{36,0617} N_{p h}
$$

Плотность фононного газа определяется из выражения для потока в гелии II. Поток определяется импульсом в системе отсчета, движущейся со сверхтекучей компонентой:

$$
\vec{p}=\vec{j}-\rho \overrightarrow{\mathrm{v}}_{s}=\rho_{n} \overrightarrow{\mathrm{v}}_{n}+\rho_{s} \overrightarrow{\mathrm{v}}_{s}-\left(\rho_{n}+\rho_{s}\right) \overrightarrow{\mathrm{v}}_{s}=\rho_{n}\left(\overrightarrow{\mathrm{v}}_{n}-\overrightarrow{\mathrm{v}}_{s}\right)=\rho_{n} \vec{w}
$$

где $\vec{w}=\overrightarrow{\mathrm{v}}_{n}-\overrightarrow{\mathrm{v}}_{s}$ - относительная скорость нормальной и сверхтекучей компоненты [9].

Этот же импульс по определению равен:

$$
\rho_{n p h} \vec{w}=\frac{1}{(2 \pi \hbar)^{3}} \int_{0}^{\infty} \int_{0}^{\pi} \int_{0}^{2 \pi}\left(e^{\frac{c p-\vec{p} \vec{w}}{k T}}-1\right)^{-1} \vec{p} p^{2} d p \sin (\vartheta) d \vartheta d \varphi .
$$

Для малых значений разности скоростей $|\vec{w}|$ функцию распределения Планка в (7) можно разложить в ряд по $\vec{p} \vec{w}$, и удержать только второй член разложения, т.к первый член разложения ввиду несимметричной подынтегральной функции в симметричных пределах интегрирования равен нулю:

$$
\int_{-\infty}^{\infty} n(c p) \vec{p} d \vec{p}=\int_{-\infty}^{\infty} n(c p)\left(p_{x} \vec{e}_{x}+p_{y} \vec{e}_{y}+p_{z} \vec{e}_{z}\right) d p_{x} d p_{y} d p_{z}=0 .
$$

Для вычисления второго члена разложения (7) поступаем следующим образом. Умножаем (7) скалярно слева и справа на $\vec{w}$. В итоге имеем:

$$
\rho_{n p h}|\vec{w}|^{2}=\left.\frac{-1}{(2 \pi \hbar)^{3}} \int_{0}^{\infty} \int_{0}^{\pi} \int_{0}^{2 \pi} \frac{d}{d(c p)}\left(\left(e^{\frac{c p-\vec{p} \vec{w}}{k T}}-1\right)^{-1}\right)\right|_{\vec{p} \vec{w}=0}(\vec{p} \vec{w})^{2} p^{2} d p \sin (\vartheta) d \vartheta d \varphi
$$


В правой части (9) произведение $\vec{p} \vec{w}$ в сферической системе импульсов имеет вид: $\vec{p} \vec{w}=p|w| \sin (\vartheta) \cos (\varphi)$.

Тогда, после сокращения общего множителя $|w|^{2}$, выражение (9) принимает вид:

$$
\begin{aligned}
& \rho_{n p h}=-\frac{1}{(2 \pi \hbar)^{3}} \frac{1}{c} \int_{0}^{\infty} \int_{0}^{\pi} \int_{0}^{2 \pi} \frac{d n(c p)}{d p} p^{4} \sin ^{3}(\vartheta) \cos ^{2}(\varphi) d p d \vartheta d \varphi= \\
& =-\frac{1}{(2 \pi \hbar)^{3}} \frac{1}{c} \int_{0}^{\infty} p^{4} d(n(c p)) \int_{0}^{\pi} \sin ^{3}(\vartheta) d \vartheta \int_{0}^{2 \pi} \cos ^{2}(\varphi) d \varphi= \\
& =-\frac{1}{(2 \pi \hbar)^{3}} \frac{1}{c} \int_{0}^{\infty} p^{4} d(n(c p)) \frac{\pi^{\frac{1}{2}} \Gamma(2)}{\Gamma\left(\frac{3}{2}+1\right)} \pi=-\frac{1}{(2 \pi \hbar)^{3}} \frac{4 \pi}{3} \frac{1}{c}\left(\left.n(c p) p^{4}\right|_{0} ^{\infty}-4 \int_{0}^{\infty} n(c p) p^{3} d p\right)= \\
& =\frac{4}{3} \frac{E_{p h}}{c^{2}} .
\end{aligned}
$$

Таким образом, существующие представления о фононной составляющей нормальной компоненты жидкого гелия II адекватно описываются функцией распределения Планка для частиц с энергией $\varepsilon(p)$.

\section{СВОБОДНАЯ ЭНЕРГИЯ, ЭНЕРГИЯ, ЧИСЛО РОТОНОВ И РОТОННАЯ ЧАСТЬ ПЛОТНОСТИ НОРМАЛЬНОЙ КОМПОНЕНТЫ ГЕЛИЯ ІІ}

В работе [1] приведены результаты расчетов для плотности ротонов, в которых в функция распределения Планка энергия ротонов представлена в виде:

$$
\varepsilon_{r}(\vec{p})=\Delta+\frac{\left(\vec{p}-\vec{p}_{0}\right)^{2}}{2 \mu}
$$

где $\Delta$ - энергетическая ротонная щель, $\vec{p}_{0}$ - импульс минимума $\varepsilon(\vec{p}), \mu$ - эффективная масса ротона.

Выражение (11) указывает на существование в нормальной компоненте гелия II направленного движения с импульсом $\vec{p}_{0}$.

На самом деле весь объем жидкого гелия II находится в равновесии, и любые направленные движения отсутствуют. Это означает, что наряду с кинетической энергией движения ротона в направлении $\vec{p}_{0}$, равной $\frac{\left(\vec{p}-\vec{p}_{0}\right)^{2}}{2 \mu}$, должна присутствовать кинетическая энергия движения ротона в направлении $-\vec{p}_{0}$, равная $\frac{\left(\vec{p}+\vec{p}_{0}\right)^{2}}{2 \mu}$. В связи с этим энергия ротонной компоненты должна равняться средней энергии направленных навстречу потоков ротонов:

$$
\varepsilon_{r}(\vec{p})=\frac{\left(\vec{p}-\vec{p}_{0}\right)^{2}}{4 \mu}+\frac{\left(\vec{p}+\vec{p}_{0}\right)^{2}}{4 \mu}=\Delta_{0}+\frac{p^{2}}{2 \mu}
$$

где $\Delta_{0}=\frac{p_{0}^{2}}{2 \mu}$ - ротонная щель, определяемая импульсом ротона.

Цифра 4 в знаменателе (12) необходима для обеспечения перехода к классической записи зависимости энергии от импульса при $\vec{p}_{0} \rightarrow 0$.

О возможности существования встречного движения атомов в гелии II будет сказано ниже.

Полагаем $\Delta_{0}>k T$. Тогда функция распределения Планка может быть преобразована в функцию Больцмана. В этих условиях свободная энергия единичного объема ротонной компоненты имеет вид:

$$
\begin{aligned}
& F_{r}(T)=-\frac{k T}{(2 \pi \hbar)^{3}} \int_{0}^{\infty} \int_{0}^{\pi} \int_{-\pi}^{\pi} \ln \left(1+n\left(\varepsilon_{r}\right)\right) d \vec{p} \approx \\
& \approx-\frac{k T}{(2 \pi \hbar)^{3}} \int_{0}^{\infty} \int_{0}^{\pi} \int_{-\pi}^{\pi} p^{2} d p \cdot \sin (\vartheta) d \vartheta d \varphi \cdot \exp \left(-\frac{p^{2}}{2 \mu k T}-\frac{\Delta_{0}}{k T}\right)=-k T\left(\frac{\mu k T}{2 \pi \hbar^{2}}\right)^{\frac{3}{2}} e^{-\frac{\Delta_{0}}{k T}} .
\end{aligned}
$$

Выражение (13) впервые получено Ландау[1].

Количество ротонов в единичном объеме вычисляется аналогично (13), и определяется выражением: 


$$
N_{r}(T)=\frac{e^{-\frac{\Delta_{0}}{k T}}}{(2 \pi \hbar)^{3}} \int_{0}^{\infty} \int_{0}^{\pi} \int_{-\pi}^{\pi} \exp \left(-\frac{p^{2}}{2 \mu k T}\right) p^{2} d p \cdot \sin (\vartheta) d \vartheta d \varphi=\left(\frac{\mu k T}{2 \pi \hbar^{2}}\right)^{\frac{3}{2}} e^{-\frac{\Delta_{0}}{k T}}
$$

Таким образом, свободная энергия единичного объема ротонов нормальной компоненты гелия II связана с количеством ротонов выражением $F_{r}(T)=-k T N_{r}(T)$.

Ротонная часть плотности нормальной компоненты гелия II определяется из выражения (7). Ее величина равна:

$$
\rho_{n r} \vec{w}=\frac{e^{-\frac{\Delta_{0}}{k T}}}{(2 \pi \hbar)^{3}} \int_{0}^{\infty} \int_{0}^{\pi} \int_{0}^{2 \pi} \vec{p} p^{2} d p \sin (\vartheta) d \vartheta d \varphi \cdot \exp \left(-\frac{p^{2}}{2 \mu k T}+\frac{\vec{p} \vec{w}}{k T}\right)
$$

Разлагая экспоненту в ряд по малым $|\vec{w}|$, получим более простое выражение для определения ротонной части нормальной плотности:

$$
\rho_{n r} \vec{w} \approx \frac{e^{-\frac{\Delta_{0}}{k T}}}{(2 \pi \hbar)^{3}} \frac{1}{k T} \int_{0}^{\infty} \int_{0}^{\pi} \int_{0}^{2 \pi} \vec{p}(\vec{p} \vec{w}) p^{2} d p \sin (\vartheta) d \vartheta d \varphi \cdot \exp \left(-\frac{p^{2}}{2 \mu k T}\right) .
$$

Умножаем (16) скалярно слева и справа на вектор $\vec{w}$. В итоге имеем:

$$
\rho_{n r}|\vec{w}|^{2} \approx \frac{e^{-\frac{\Delta_{0}}{k T}}}{(2 \pi \hbar)^{3}} \frac{1}{k T} \int_{0}^{\infty} \int_{0}^{\pi} \int_{0}^{2 \pi}(\vec{p} \vec{w})^{2} p^{2} d p \sin (\vartheta) d \vartheta d \varphi \cdot \exp \left(-\frac{p^{2}}{2 \mu k T}-\frac{\Delta_{0}}{k T}\right) .
$$

Подстановка $\vec{p} \vec{w}$ в сферической системе импульсов в виде $\vec{p} \vec{w}=p|w| \sin (\vartheta) \cos (\varphi)$, и последующее интегрирование (17) дает: $\rho_{n r}=\mu N_{r}$.

Таким образом, Число ротонов $N_{r}$, а с ним и $\rho_{n r}$ зависят от температуры экспоненциально.

Представленные выше расчеты свободной энергии, энергии и числа квазичастиц в единичном объеме нормальной компоненты гелия II соответствуют первоначально полученным Ландау для равновесного распределения ротонов (12) [1].

Затем, немного позже он изменил энергетический спектр на неравновесный (11), добавив вместе с тем вопрос о причине появления неравновесности.

Таким образом, исходя из вышеизложенного можно сделать вывод, что отмеченное выше расхождение в экспериментальном определении величины ротонной энергетической щели, импульса и эффективной массы (таблица 1), а также отсутствие описания физической природы сил, удерживающих атомы гелия в ротоне, указывает на необходимость поиска моделей описания физической природы и структуры ротона.

Целью настоящей работы является описание физической модели возникновения ротона, определение его геометрических размеров и внутренней структуры, а также оценка его термодинамических параметров.

\section{РАВНОВЕСНЫЙ ЭНЕРГЕТИЧЕСКИЙ СПЕКТР РОТОНОВ}

Равновесный энергетический спектр для ротонов вида (12) возможен, если он образован объемным вихрем из движущихся атомов гелия в цилиндрическом объеме диаметром $D=D_{r}$ и высотой $h=L_{z}$. Вихревое движение атомов организовано так, что вблизи оси цилиндра они движутся, например, вдоль направления оси цилиндра (вверх), а на внешней границе цилиндра - в обратном направлении. На Рис. 1а) схематически изображено вихревое движение атомов гелия II внутри цилиндрической ячейки.

Существование цилиндрических конвективных ячеек со свободными граничными условиями в вязкой несжимаемой жидкости впервые экспериментально исследовано и теоретически описано в работе [10]. Здесь показано, что такие ячейки имеют такие геометрические размеры, что отношение диаметра ячейки $D_{r}$ к высоте $L_{z}$ постоянно, и равно $D_{r} / L_{z}=3,44$.

B такой ячейке в вертикальном сечении существуют взаимно противоположные течения жидкости: на верхней границе ячейки скорость течения жидкости направлена от центра, а на нижней - к ее центру или наоборот.

Следует отметить, что на вихревую природу ротона указывал в своей работе Фейнман [11]. Он писал о ротонах, как о вихревых кольцах, аналогичных кольцам дыма. При этом импульс вихря будет связан не с его поступательным движением как целого, а с движением вокруг цилиндрической поверхности, изогнутой в замкнутое кольцо.

Таким образом, ротон можно рассматривать как цилиндрическую конвективную ячейку. При этом использование энергетического спектра ротонов вида (12) оправдано, и позволяет считать гелий II в целом, как покоящуюся жидкость. Пространственное распределение потоков в ротоне относится к его внутренней структуре, и характеризует только его внутренние свойства. 


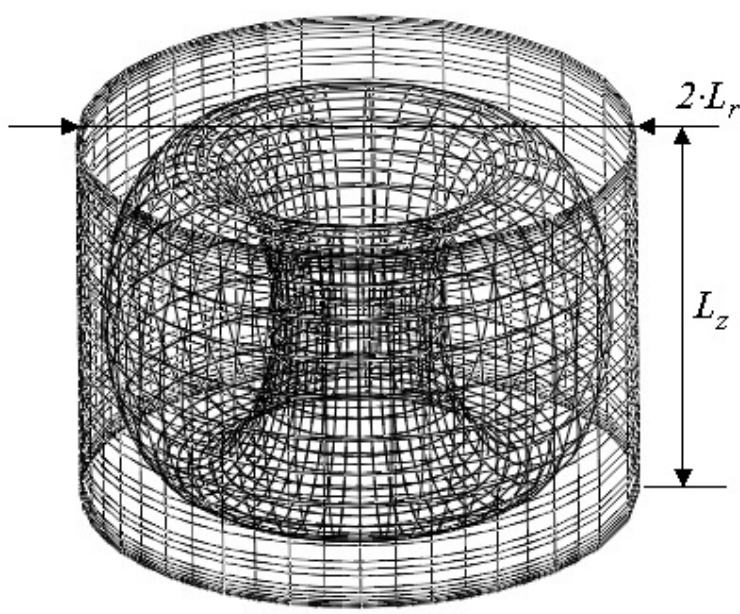

a)

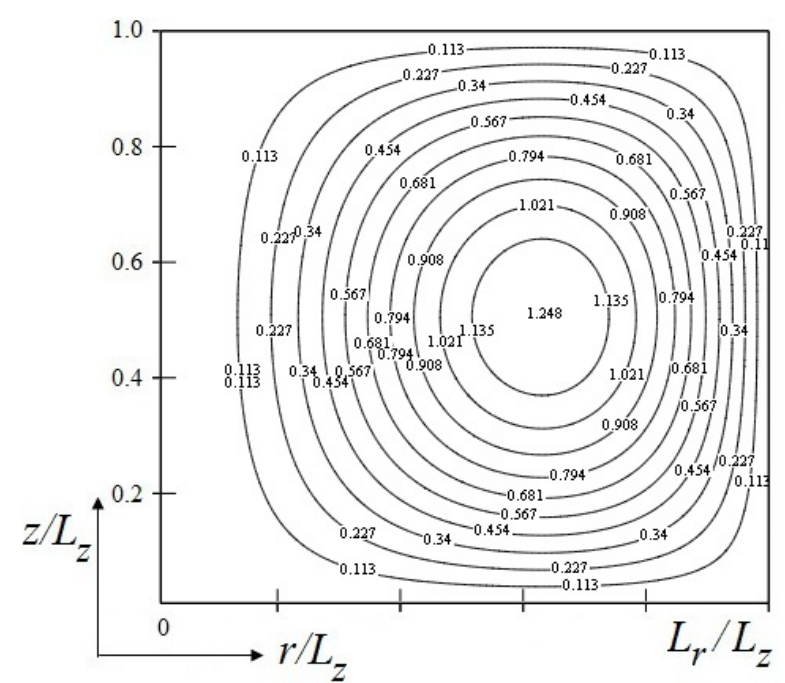

б)

Рис. 1. Схематическое изображение общего вида цилиндрической конвективной ячейки - ротона а). Распределение линий Стокса $\psi(r, z)$ внутри ротона - б)

\section{ОЦЕНКА ТЕРМОДИНАМИЧЕСКИХ ПАРАМЕТРОВ ФОРМИРОВАНИЯ РОТОНОВ В ГЕЛИИ ІІ}

Будем считать, что в гелии II ротонами являются цилиндрические конвективные ячейки. Тогда для подтверждения этого предположения следует оценить значения термодинамических параметров гелия II. Таким параметрами являются коэффициенты теплового расширения $\beta$, кинематической вязкости $v \quad$ и температуропроводности $\chi$ [12]. Эти термодинамические параметры задают величину числа Рэлея, которое, в конечном итоге, определит условия возникновения и устойчивого существования ячеек - ротонов.

Число Прандтля. Число Прандтля Рr определяется отношением $\operatorname{Pr}=v / \chi$. Как правило, для жидких сред его значение больше 1 [13].

Число Рэлея. Для устойчивого существования конвективной ячейки со свободными граничными условиями число Рэлея должно равняться минимальному числу Рэлея $R_{m}=g \beta L_{z}^{3} T_{\lambda} / v \chi=657,511$ [10,12,13].

Оценим значения размерных коэффициентов, определяющих число Рэлея.

Коэффициент теплового расширения для нормальной компоненты сверхтекучего гелия определим выражением $\beta(T)=\frac{1}{\rho_{n}} \frac{d \rho_{n}}{d T}$. В интервале температур $0,15 \leq T \leq 1,95(K)$, исходя из (10) и (17), $2,58 \leq \beta(T) \leq 25,45\left(K^{-1}\right)$, а среднее значение $\bar{\beta}=\frac{1}{1,8} \int_{0,15}^{1,95} \beta(x) d x$ равно $\bar{\beta} \approx 8,82$.

Динамический и кинематический коэффициенты вязкости определим, исходя из приведенных в научной литературе данных.

В обзоре [14] отмечено, что коэффициент динамической вязкости гелия II заведомо меньше, чем $10^{-11}$ Па·с.

Капица из своих опытов определил верхний предел вязкости сверхтекучей компоненты [15]. В предположении ламинарности течения им было получено значение коэффициента динамической вязкости $\approx 10^{-11} \cdot \Pi \mathrm{a} \cdot \mathrm{c}$.

В работе [16] значение коэффициента динамической вязкости оценивается величиной порядка $10^{-11}$ Па с. В [17] коэффициент динамической вязкости определяется величиной $\eta=10^{-14}\left(\frac{\Gamma \cdot c}{c M^{2}}\right)=10^{-14}\left(\frac{980 \cdot 2 \cdot c M \cdot c}{c M^{2} c^{2}}\right)=0,98 \cdot 10^{-12} \Pi$ а $\cdot c$.

Поэтому в дальнейших расчетах будем считать, что коэффициент динамической вязкости равен $10^{-12}$ Па·с. Отсюда коэффициент кинематической вязкости будет иметь величину порядка $v \simeq 10^{-12}(\Pi a \cdot c) / \rho\left(\frac{\kappa 2}{\mu^{3}}\right) \simeq 6,7 \cdot 10^{-15} \mathrm{M}^{2} \mathrm{c}^{-1}$.

Для указанного выше диапазона изменения коэффициента теплового расширения $\beta(T)$, число Прандтля находится в интервале $\operatorname{Pr}=3,25 \ldots 3,83$ [18].

Из приведенных выше оценок следует, что, например, при $\bar{\beta}=11,2, \operatorname{Pr}=3,4988313$ число Рэлея 
удовлетворяет условию устойчивого существования цилиндрической конвективной ячейки:

$$
R=3,4988313 \cdot \frac{11,2\left(\frac{1}{K}\right) \cdot 10\left(\frac{M}{c^{2}}\right) \cdot(3,262(M))^{3} \cdot 2,17(K)}{(6,7)^{2}\left(\frac{M^{4}}{c^{2}}\right)} \approx 657,511 .
$$

Таким образом, приведенные выше значения термодинамических параметров гелия II соответствуют тем, которые необходимы для формирования устойчивой конвективной ячейки.

\section{ПРИРОДА И ВНУТРЕННЕЕ СТРОЕНИЕ РОТОНА}

До настоящего времени природа возникновения и физические параметры ротонов не установлены. Поэтому ниже будет предложено описание вышеприведенных характеристик этой квазичастицы.

Но, перед описанием природы и внутреннего строения ротона, полезно проследить процесс перехода жидкого гелия в сверхтекучее состояние.

Для понижения температуры жидкого гелия ниже $\lambda$ - точки необходимо производить постоянную откачку паров гелия из криостата. Достижение определенной равновесной температуры, находящейся ниже $\lambda$ - точки, обеспечивается длительным по времени поддержанием определенного пониженного давления в гелиевом криостате [3].

В процессе установления термодинамического равновесия при понижении давления в объеме гелия II возможна реализация горизонтально-многослойной структуры пространственного распределения ротонов.

Опишем более подробно процесс формирования такого пространственного распределения ротонов.

\section{Горизонтально-многослойная структура пространственного распределения ротонов}

Горизонтально-многослойная структура пространственного распределения ротонов, по-видимому, возникает в связи с формированием на верхней границе гелия, при переходе через $\lambda$ - точку, одноатомного слоя сверхтекучей жидкости. В этих условиях, по-видимому, находящаяся снизу нормальная компонента гелия II образует горизонтальный переходной слой, толщина которого достаточно мала, и сопоставима с величиной межатомного расстояния в гелии II ( $L_{0 z} \simeq 3,579 \AA$ ). В этом переходном слое температура нормальной компоненты гелия II будет нарастать в вертикальном направлении от нескольких градусов К на нижней границе слоя (при малых отклонениях температуры от $\lambda$ - точки) до нуля на верхней границе слоя.

Таким образом, исходя из описанных выше условий, мы приходим к задаче Рэлея-Бенара о конвекции в слое подогреваемой снизу вязкой, несжимаемой жидкости [10].

В указанных выше условиях при свободных границах в переходном слое нормальной компоненты гелия II будут возникать цилиндрические конвективные ячейки (читай ротоны) с отношением диаметра к высоте порядка $D_{r} / h=3,44$ [10]. Схематический вид такой цилиндрической ячейки и линии Стокса $\psi(r, z)=\operatorname{Arsin}\left(\frac{\pi z}{L_{z}}\right) J_{1}\left(\frac{\sigma_{1,1} r}{L_{r}}\right)$ приведены на Рис. 1. В такой ячейке атомы гелия движутся по тороидальной поверхности (Рис. 1, a)) с возмущенной скоростью $\overrightarrow{\mathrm{v}}(r, z)$, и соответствующей ей возмущенной температурой $T(r, z)$ :

$$
\begin{aligned}
& \mathrm{v}_{z}(r, z)=A \sin \left(\frac{\pi z}{L_{z}}\right) J_{0}\left(\frac{\sigma_{1,1} r}{L_{r}}\right), \\
& \mathrm{v}_{r}(r, z)=-A \frac{\pi}{L_{z}} \frac{L_{r}}{\sigma_{1,1}} \cos \left(\frac{\pi z}{L_{z}}\right) J_{1}\left(\frac{\sigma_{1,1} r}{L_{r}}\right), \\
& T(r, z)=B \sin \left(\frac{\pi z}{L_{z}}\right) J_{0}\left(\frac{\sigma_{1,1} r}{L_{r}}\right),
\end{aligned}
$$

где $r, z$ - горизонтальная и продольная координаты соответственно, $\mathrm{V}_{z}(r, z), \mathrm{v}_{r}(r, z)$ - вертикальная и горизонтальная проекции возмущенной скорости атомов гелия соответственно, $A, B$ - константы, $J_{k}(x)$ функции Бесселя первого рода $k$ - го порядка от аргумента $x, \sigma_{1,1}=3,832$ - первый ноль функции Бесселя первого рода первого порядка $\left(J_{1}\left(\sigma_{1,1}\right)=0\right)$.

Тороидальная поверхность внутри цилиндра (Рис. 1, а)) соответствует определенному значению линии Стокса (рис. 1б)), которая определяется через горизонтальную проекции возмущенной скорости движения вещества в конвективной ячейке соотношением $\mathrm{v}_{z}(r, z)=r^{-1} \partial \psi(r, z) / \partial r$. 
Из (19) следует, что на верхней границе ячейки $z=L_{z}$ горизонтальная скорость максимальна при $r=\sigma_{m} L_{r} / \sigma_{1,1}$, где $\sigma_{m}=1,8411$ - аргумент, при котором функция $J_{1}(x)$ принимает максимальное значение: $J_{1}\left(\sigma_{m}\right)=0,582$.

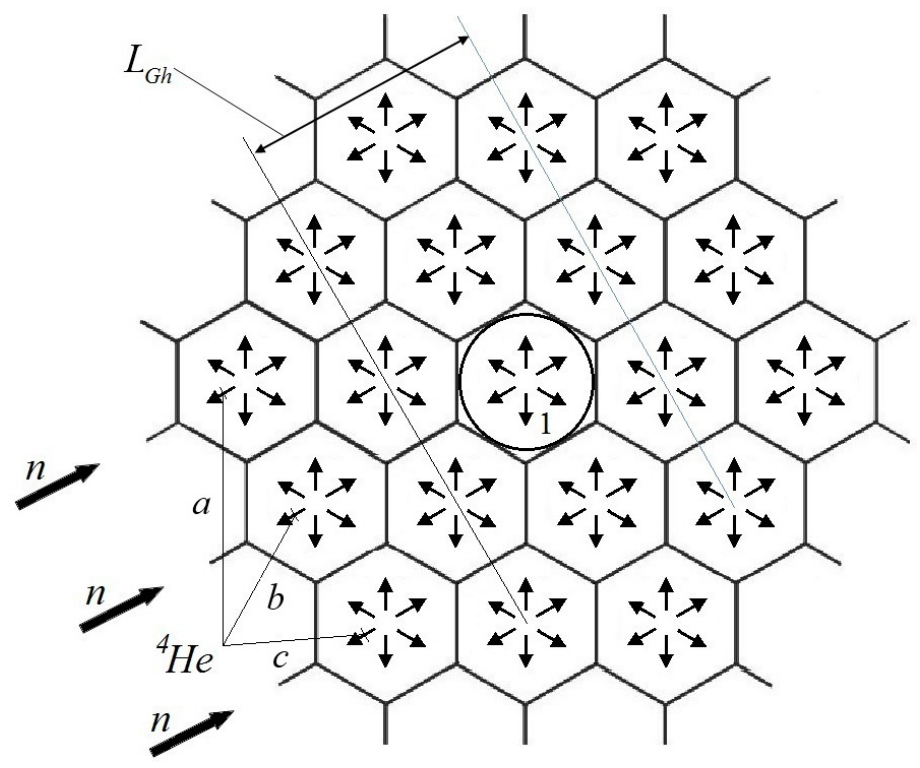

Рис. 2. Горизонтальное сечение слоя ротонов на его верхней границе с указанием направления движением атомов гелия (от центра ячейки к ее внешним границам).

При увеличении числа цилиндрических ячеек будет наблюдаться их упаковка таким образом, что каждая ячейка будет граничить с шестью такими же. На конечной стадии упаковки цилиндрические ячейки заполнят весь объем переходного слоя таким образом, что границы между ячейками будут иметь вид ячеек Бенара, т.е. гексагонов.

Сказанное выше показано на рис. 2. Здесь цифрой 1 показан вид сверху верхней границы цилиндрической ячейки, вписанной в правильный шестиугольник. Внутри каждого шестиугольника стрелками от его центра схематично указано направление движения атомов гелия на верхней границе ячейки. На нижней границе ячейки атомы гелия движутся в противоположном направлении. В точках $a, b, c$ встречная скоростью атомов гелия максимальна, $L_{G h}$ - период гексагональной решетки.

Заполнение объема гелия II нижележащим слоем ротонов будет происходить следующим образом.

После формирования первого слоя конвективных ячеек - ротонов, снизу, на глубине межатомного расстояния в гелии II снова начнет формироваться мономолекулярный слой сверхтекучей жидкости. Такое перемещение сверхтекучей жидкости вглубь гелия II можно объяснить следующим образом. При понижении температуры верхней границы гелия II, к последней будет направлен поток нормальной (теплой) компоненты гелия II. Но согласно теории второго звука, нормальная и сверхтекучая компоненты перемещаются навстречу друг другу, обеспечивая отсутствует суммарного потока вещества [1]. Поэтому длительное охлаждение верхней границы гелия II будет способствовать такому же по длительности проникновению сверхтекучей компоненты вглубь, т.к. временные периоды колебаний компонент гелия II одинаковые.

Таким образом, снизу первого слоя конвективных ячеек, в результате описанного выше механизма перемещения сверхтекучей компоненты, снова образуется мономолекулярный слой сверхтекучей компоненты гелия II. При этом сверхтекучая и нормальная компоненты гелия II характеризуются соизмеримыми геометрическими размерами расслоения.

После образования мономолекулярного слоя сверхтекучей компоненты, находящаяся снизу него нормальная компонента гелия II снова образует горизонтальный переходной слой толщиной порядка межатомного расстояния в гелии II. В этом новом переходном слое начнет формироваться второй слой ротонов. После завершения формирования второго слоя ротонов сверхтекучая компонента снова начнет накапливаться снизу второго слоя, и процесс формирования третьего и последующих слоев конвективных ячеек - ротонов повторится.

Такой послойный процесс формирования ротонного газа будет осуществляться до наступления термодинамического равновесия, и накопления отвечающего данной температуре (давлению насыщенных паров) количества ротонов.

Исходя из характерных размеров элементарной конвективной ячейки со свободными границами, область расположения ротона в нормальной компоненте гелия II представляет гексагональную ячейку с характерными размерами: $L_{z}=3,262 \AA, L_{r}=1,72 \cdot L_{z}=5,61 \AA$ [10], где высота гексагональной ячейки $L_{z}$ выбрана исходя из 
величины волнового числа, приходящегося на ротонный минимум.

Таким образом, из рис. 2 следует, что в гелии II образуется гексагональная кристаллическая решетка с периодом $L_{z}=3,262 \AA$ в вертикальном направлении, и $L_{G h}=2 \cdot D_{r} \cdot 2 / \sqrt{3}=25,944 \AA$ - в горизонтальном.

На рис. 2 схематично показано горизонтальное сечение верхней границы слоя ротонов. Стрелками показано движение атомов гелия из центра ячеек на периферию. Приведенная на рис.2 гексагональная структура верхней границы конвективных ячеек повторяется вглубь рисунка с периодом $L_{z}$.

Наличие описанной выше кристаллической структуры расположения ротонов приводит к рассеянию нейтронов не на ротонах, как считалось ранее, а на атомах гелия, из которого образованы ротоны.

На рис. 2. линиями показаны точки верхней границы ячейки, где нейтроны $n$ рассеиваются на атомах гелия ${ }^{4} \mathrm{He}$, движущихся навстречу нейтронам. В этих точках атомы гелия имеют максимальную скорость $-V_{\text {He }}[10]$. В точках, расположенных симметрично относительно центра цилиндрической ячейки, максимальная скорость атомов гелия равна $V_{H e}$, и направлена вдоль скорости нейтронов.

Расстояние указанных точек от центра ячейки равно $R_{m}=\sigma_{0,1} L_{r} / \sigma_{1,1}$, где $\sigma_{0,1} \approx 2,405$ и $\sigma_{1,1} \approx 3,832-1$ - й ноль функции Бесселя первого рода нулевого и первого порядка соответственно [19].

Таким образом, из приведенного выше описания следует, что моноэнергетические холодные нейтроны рассеиваются на периодической решетке $L_{z} \times L_{G h}$, состоящей из атомов гелия, движущихся навстречу или попутно нейтронам. Максимальная встречная скорость атомов гелия равна $-V_{\text {He}}$, в попутном направлении $V_{H e}$.

\section{КВАНТОВАНИЕ ЭНЕРГЕТИЧЕСКОГО СПЕКТРА РОТОНОВ}

Отсутствие в объеме гелия II направленных потоков (беспотоковый энергетический спектр) позволяет представить энергию ротонов в классическом виде:

$$
\varepsilon_{r}\left(p_{r,} p_{z}\right)=\frac{(\vec{p})^{2}}{2 \mu} .
$$

Ввиду отмеченной выше пространственной периодичности распределения ротонного газа, энергетический спектр ротонов (20) должен квантоваться по вертикальному $p_{z}$ и $p_{r}$ горизонтальному импульсам. Такое квантование спектра (20) можно представить в виде:

$$
\varepsilon_{r, n m}\left(p_{r}, p_{z}\right)=\frac{\left(p_{z}-n p_{z 0}\right)^{2}+\left(p_{r}-m p_{r 0}\right)^{2}}{2 \mu}
$$

где $m=1,2,3 \ldots$ и $n=m-1$ - числа квантования, $p_{z 0} / \hbar=2 \pi / L_{z} \AA^{-1}, p_{r 0} / \hbar=2 \pi / L_{G h} \AA^{-1}$.

Реальные значения параметров в выражении (21) можно установить исходя из экспериментальных данных по рассеянию холодных нейтронов на ротонах. Для этого используем данные зависимости энергетического спектра ротонов от волнового числа, которые сведены в одну кривую в работе [20]. Исходя из вида этой кривой можно сделать вывод, что энергетический спектр ротонов квантуется по волновому числу и энергии.

На рис. 3 приведен энергетический спектр элементарных возбуждений в гелии II как функция волнового числа для температур ниже $\lambda$ - точки. На этом рисунке экспериментальные точки из различных источников отмечены $1,2,3,4,5[20]$.

Обработка приведенных данных позволяет сформулировать следующие выводы относительно параметров квантования энергетического спектра ротонов (21):

- в спектре наблюдается квантование по волновым числам $p_{z}$ и $p_{r}$ с постоянными $p_{z 0} / \hbar=1,925 \AA^{-1}$ и $p_{r 0} / \hbar=0,242 \AA^{-1}$ соответственно;

- в спектре наблюдается квантование по энергии с постоянной $\Delta_{n}=\left((n+1) p_{r 0}\right)^{2} / 2 \mu$, где $n=0,1,2$. Такое квантование подтверждается аппроксимирующими параболами: $\varepsilon_{r, 01}\left(0, p_{z}\right) ; \varepsilon_{r, 12}\left(0, p_{z}\right) ; \varepsilon_{r, 23}\left(0, p_{z}\right)$, вид которых в определенном диапазоне импульсов хорошо описывает экспериментальную зависимость;

Количественные оценки по экспериментальным данным, приведенным на рис. 3, для числа квантования $n=1$ дают следующие значения массы ротона и ширины энергетической ротонной щели: $\mu=0,1649 \cdot m_{H e}$; $\Delta_{1}=8,606 \mathrm{~K}$.

Следует отметить, что полученные значения массы ротона и ширины энергетической ротонной щели соответствуют приведенным в таблице 1.

Сравнение экспериментальных данных и предложенной модели указывают на квантование энергетического спектра ротонов из-за их периодического расположения в горизонтальном и вертикальном направлениях в объеме гелия II.

В предложенной модели в ротонной части спектра энергия ротонов представлена в классическом 
виде (20). Суммирование энергий двух ротонов дает энергию, постулируемую Ландау для обоснования появления ротонного минимума:

$$
\begin{aligned}
& \bar{\varepsilon}_{r, 21}\left(0, p_{z}\right)=\frac{\varepsilon_{r, 21}\left(0, p_{z}\right)+\varepsilon_{r, 21}\left(0,-p_{z}\right)}{2}=\frac{\left(p_{z}-2 p_{z 0}\right)^{2}+\left(p_{z}+2 p_{z 0}\right)^{2}+2\left(p_{r 0}\right)^{2}}{4 \mu}= \\
& =\frac{\left(p_{z}\right)^{2}+\left(2 p_{z 0}\right)^{2}+\left(p_{r 0}\right)^{2}}{2 \mu}=\Delta_{21}+\frac{\left(p_{z}\right)^{2}}{2 \mu}
\end{aligned}
$$

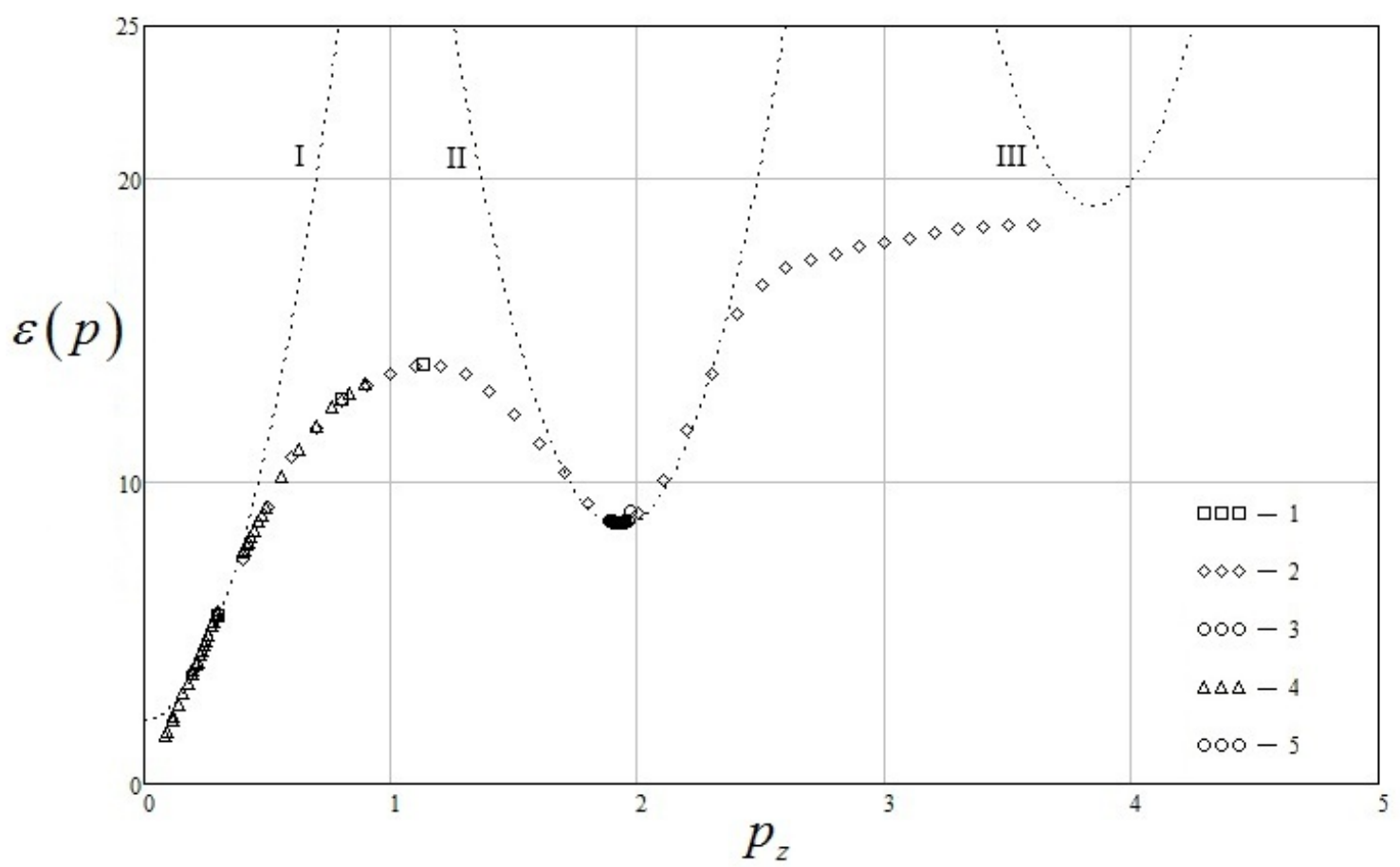

Рис. 3. Энергия $\varepsilon$ (в градусах $K$ ) элементарных возбуждений гелия II как функция импульса $p_{z}$ (в $\left.\AA^{-1}\right)[18]$.

Параболы, изображенные точками и отмеченные цифрами I, II, III соответствуют следующим числам квантования: I - $n=0, m=1$; II - $n=1, m=2$ : III - $n=2, m=3$.

Таким образом, в настоящем разделе показано, что экспериментальная зависимость энергии квазичастиц нормальной компоненты гелия II от импульса в коротковолновой области спектра может быть объяснена беспотоковым видом энергетического спектра ротонов и их периодическим расположением в горизонтальном и вертикальном направлениях.

\section{ПЛОТНОСТЬ НОРМАЛЬНОЙ КОМПОНЕНТЫ ГЕЛИЯ ІІ С БЕСПОТОКОВЫМ ЭНЕРГЕТИЧЕСКИМ СПЕКТРОМ РОТОНОВ}

Первоначально, используя классическое представление зависимости энергии ротонов от импульса вида (20), Ландау рассчитал следующие термодинамические величины нормальной компоненты гелия II: свободную энергию, энтропию, теплоемкость (на единицу массы) и плотность [8]. Затем, в той же работе, повторно опубликованной в УФН, но уже с Приложением, Ландау пересчитал полученные ранее термодинамические величины для энергии ротонов, постулируемой в виде [1]:

$$
\varepsilon_{r}(p)=\Delta+\frac{\left(p-p_{0}\right)^{2}}{2 \mu} .
$$

Поэтому существует закономерный вопрос о правомерности применения зависимости энергии ротонов от импульса в классическом виде (20) или в виде (22).

Отношение плотности нормальной компоненты гелия II к полной плотности жидкого гелия (по традиции назовем это соотношение термином роэнкро) имеет вид [1]:

$$
\frac{\rho_{n}}{\rho}=\frac{16}{45} \frac{\pi^{5}}{c^{2}} \frac{k T}{\rho}\left(\frac{k T}{2 \pi \hbar c}\right)^{3}+\frac{\mu N_{r}}{\rho} .
$$

Для решения поставленного вопроса в выражение (23) будем подставлять количество ротонов либо в виде 
$N_{r}=N_{r}^{(1)}=\left(\frac{\mu k T}{2 \pi \hbar^{2}}\right)^{\frac{3}{2}} e^{-\frac{\Delta_{0}}{k T}}$, которое определяется энергией ротона в виде (20) (линия $I$ на рис. 4), либо $N_{r}=N_{r}^{(2)}=\frac{2 \mu^{\frac{1}{2}} p_{0}^{4}}{3\left(2 \pi \hbar^{2}\right)^{\frac{3}{2}}(k T)^{\frac{1}{2}}} e^{-\frac{\Delta_{0}}{k T}}$, определяемое энергией ротона в виде (22) (линия II на рис. 4).

Ответом на поставленный вопрос будет служить сравнительная оценка совпадения теоретической зависимости роэнкро от температуры двух видов энергии ротонов (20) и (22) с экспериментальными точками. При этом необходимо учитывать, что аналитические выражения зависимости роэнкро от температуры неприменимы для температур вблизи $\lambda$ - точки и в окрестности нуля [9].

Для ответа на поставленный вопрос воспользуемся экспериментальными данными зависимости роэнкро от температуры [20].

На рис. 4 маркерами "х" приведены рекомендуемые к использованию данные экспериментальных измерений величины роэнкро в зависимости от температуры в диапазоне температур от $0,15 K$ до $1,95 K$.

Кривые $I$ и $I I$ построены, исходя из выражения (23) для количества ротонов в единичном объеме вида (20) и (22) соответственно:

$$
\begin{aligned}
& \left(\frac{\rho_{n}}{\rho}\right)_{I}=A_{I} x^{4}+B_{I} x^{1.5} \exp \left(-\frac{D_{I}}{x}\right) \\
& \left(\frac{\rho_{n}}{\rho}\right)_{I I}=A_{I I} x^{4}+B_{I I} x^{-0.5} \exp \left(-\frac{D_{I I}}{x}\right)
\end{aligned}
$$

где $x=T /(1 K)$ - безразмерная температура; $A_{I}=1,19 \cdot 10^{-4}, \quad B_{I}=5,66, \quad D_{I}=6,7879 ; \quad A_{I I}=1,21 \cdot 10^{-4}$, $B_{I I}=204,32, D_{I I}=11,152$ - численно подобранные оптимальные значения констант в (24), (25).

Подбор оптимальных параметров осуществлялся по минимальному среднеквадратичному отклонению теоретической зависимости от экспериментальных точек.

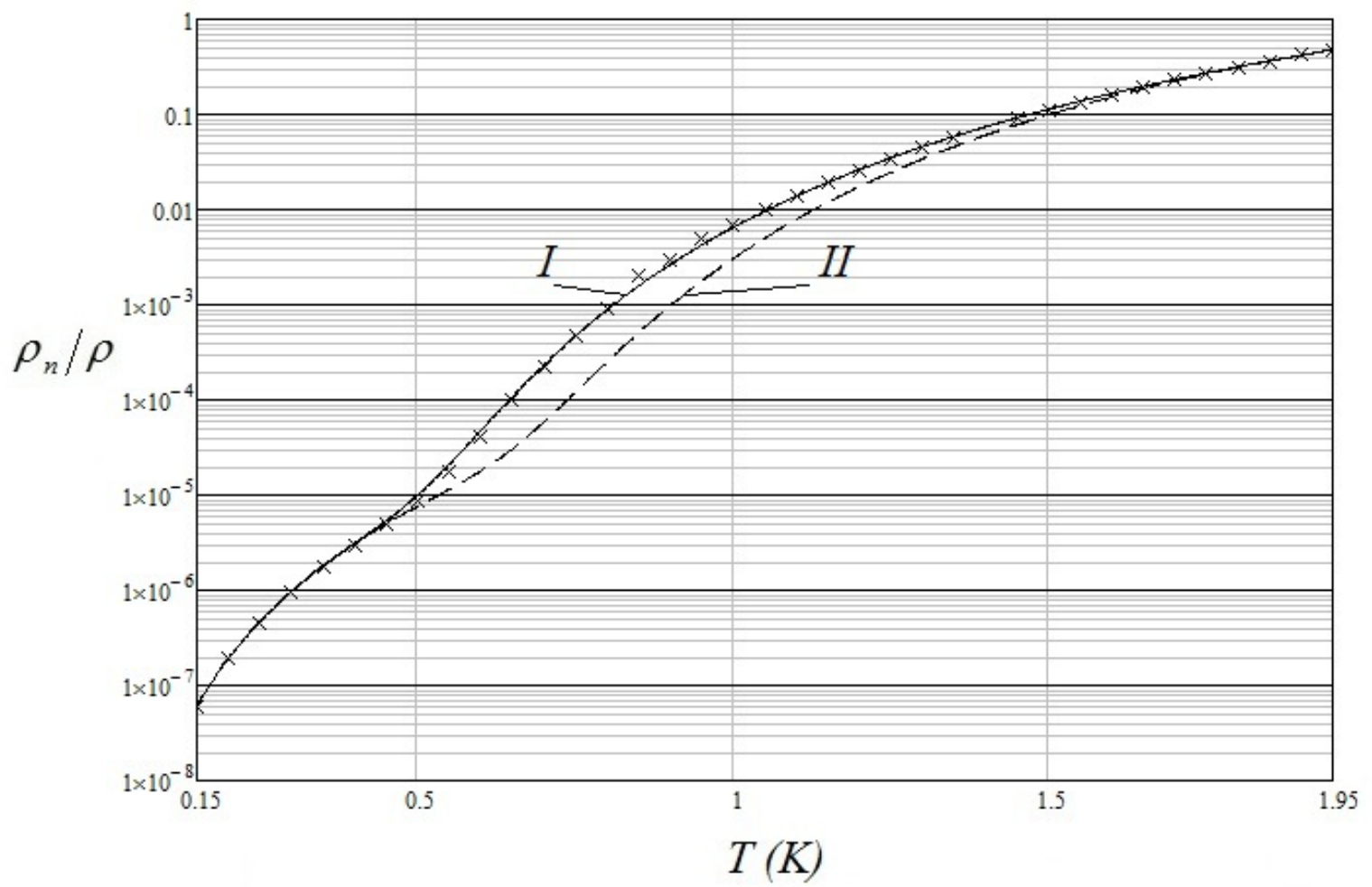

Рис. 4. Зависимость роэнкро ( $\left.\rho_{n} / \rho\right)$ от температуры $T$ в диапазоне температур от $0,15 K$ до $1,95 K$.

Значками "х" отмечены экспериментально измеренные точки. Кривая $I$ соответствует $N_{r}^{(1)}$, Кривая $I I-N_{r}^{(2)}$.

Наименьшее среднеквадратичное отклонение кривой $I$ от экспериментальных точек составляет величину $1,772 \cdot 10^{-3}$, в то время, как для кривой $I I$ оно составляет величину в 2,48 раза больше $-4,395 \cdot 10^{-3}$.

Таким образом, в рассматриваемом диапазоне температур роэнкро гелия II с наибольшей степенью точности определяется беспотоковым энергетическим спектром ротонов вида (20). 


\section{УПРУГОЕ РАССЕЯНИЕ НЕЙТРОНОВ НА ДВИЖУЩИХСЯ АТОМАХ ГЕЛИЯ}

В отличие от общепринятой концепции о рассеянии медленных нейтронов на гелии II, как неупругое их рассеянии на ротонах, представим другую картину такого процесса. Будем считать, что в конвективных ячейках нейтроны упруго рассеиваются на движущихся навстречу им атомах гелия. Рассеяние нейтронов на попутных атомах гелия не рассматриваем, т.к. они замедляются и не регистрируются времяпролетным нейтронным спектрометром [3].

Для подтверждения справедливости этого предположения сравним экспериментальные данные, полученные в Стокгольмском эксперименте на времяпролетном нейтронном спектрометре, с результатами теоретического анализа, который приведен ниже.

Рассчитаем параметры рассеяний нейтрона на движущемся атоме гелия.

Определим угол упругого рассеяния нейтрона $\psi$ на движущемся в цилиндрической конвективной ячейке атоме ${ }^{4} \mathrm{He}$. Пусть атом гелия до столкновения имеет максимальную горизонтальную скорость $\vec{V}_{H e, \pm}=\mp V_{H e} \vec{V}_{n} /\left|\vec{V}_{n}\right|$ в диаметрально противоположных точках верхней границы ячейки (рис. 1,2$)$.

Масса нейтрона равна $m_{n}=1,6749287276 \cdot 10^{-24}$ г [21], что в 0,252 раза меньше массы атома гелия. Полагаем, что скорость нейтрона до столкновения равна $\vec{V}_{n}$.

В результате упругого столкновения нейтрон приобретет скорость $\vec{V}_{n 1}$, а атом гелия - $\vec{V}_{H e 1, \pm}$. При упругом столкновении, и в отсутствие внешних сил из законов сохранения импульса и кинетической энергии нетрудно получить выражение для определения относительной скорости рассеянного нейтрона $V_{n 1} / V_{n}$ :

$$
\left(\frac{V_{n 1}}{V_{n}}\right)^{2}-2 \frac{V_{n 1}}{V_{n}} \frac{1 \mp \xi_{ \pm}}{1+\zeta} \cos (\psi)+\frac{1 \mp 2 \xi_{ \pm}-\zeta}{1+\zeta}=0,
$$

где $\xi_{ \pm}=\zeta \frac{V_{H e, \pm}}{V_{n}}, \zeta=\frac{m_{H e}}{m_{n}}, \psi$ - угол рассеяния нейтрона.

В уравнении (26) верхний знак соответствует рассеянию нейтрона на движущемся навстречу, нижний - на движущемся в том же направлении атоме гелия.

Решения уравнений (26) имеют вид:

$$
\begin{gathered}
\left(\frac{V_{n 1}}{V_{n}}\right)_{1 \pm}=\frac{1 \mp \xi_{ \pm}}{1+\zeta} \cos (\psi)+\sqrt{\left(\frac{1 \mp \xi_{ \pm}}{1+\zeta}\right)^{2} \cos ^{2}(\psi)-\frac{1 \mp \xi_{ \pm}-\zeta}{1+\zeta}} \\
\left(\frac{V_{n 1}}{V_{n}}\right)_{2 \pm}=\frac{1 \mp \xi_{ \pm}}{1+\zeta} \cos (\psi)-\sqrt{\left(\frac{1 \mp \xi_{ \pm}}{1+\zeta}\right)^{2} \cos ^{2}(\psi)-\frac{1 \mp \xi_{ \pm}-\zeta}{1+\zeta}}
\end{gathered}
$$

Расчеты показывают, что наибольшую скорость имеют рассеянные нейтроны со скоростью атомов гелия $\left(V_{n 1} / V_{n}\right)_{1+}$. Поэтому величина смещения во времени брэгговского обрезания первичного пучка [3] при заданном угле рассеяния будет минимальной, т.к. она обратно пропорциональна скорости рассеянной частицы.

Этот вывод основан на следующих оценках.

Будем полагать начальную скорость $V_{n}$ такой же, что и скорость нейтрона после упругого рассеяния на ванадии, т.к. он рассеивает нейтроны изотропно и без изменения энергии [3]. В Стокгольмском эксперименте эта скорость оценивается величиной $V_{n}=973,71 \mathrm{~m} / \mathrm{c}[3]$.

Зададим начальную скорость нейтрона в виде $V_{n}=L / t_{0}$, где $L$ - расстояние пролета нейтронов в спектрометре, $t_{0}$ - время пролета.

Скорость нейтрона после его рассеяния на гелии II равна $V_{n 1}=L /\left(t_{0}+\Delta t\right)$, где $\Delta t$ - величина смещения во времени брэгговского обрезания первичного пучка. Отсюда из равенства $\left(V_{n 1} / V_{n}\right)_{1+}=1 /\left(1+\Delta t / t_{0}\right)$ можем оценить величину относительного смещения во времени $\Delta t / t_{0}$ :

$$
\Delta t / t_{0}=\left(\frac{V_{n 1}}{V_{n}}\right)_{1+}^{-1}-1 .
$$

Из выражения (29) и экспериментальных данных, приведенных в [3], можно определить максимальную горизонтальную встречную скорость $V_{H e,+} / V_{n}$ в конвективной ячейке рассеивающего центра (атома гелия).

На рис. 5 приведены экспериментальные точки ( о и ×), показывающие зависимость горизонтальной скорости рассеивающего нейтрон атома гелия $V_{H e,+} / V_{n}$ от угла наблюдения $\theta$. 
Таблица 2.

Максимальная горизонтальная скорость атома гелия $V_{H e,+} / V_{n}$ в конвективной ячейке при температурах $T=1,4-1,5{ }^{\circ} \mathrm{K}$ в зависимости от угла наблюдения $\theta[3]$.

\begin{tabular}{|c|c|c|c|c|c|c|c|c|}
\hline \multirow[t]{2}{*}{$\begin{array}{c}\text { Вид } \\
\text { фильтра }\end{array}$} & \multicolumn{2}{|c|}{$\begin{array}{c}\text { Угол } \\
\text { наблюдения, } \theta\end{array}$} & \multirow[t]{2}{*}{$\begin{array}{c}t_{0}, \\
\text { мкс }\end{array}$} & \multirow[t]{2}{*}{$\begin{array}{c}t_{0}+\Delta t, \\
\quad \text { мкс }\end{array}$} & \multirow[t]{2}{*}{$\begin{array}{l}\Delta t \\
\text { мкс }\end{array}$} & \multirow[t]{2}{*}{$\Delta t / t_{0}^{*)}$} & \multirow[t]{2}{*}{$\begin{array}{c}{ }^{*)} \\
\frac{V_{H e,+}}{V_{n}}\end{array}$} & \multirow[t]{2}{*}{$\left(\frac{V_{n 1}}{V_{n}}\right)_{1+}^{*)}$} \\
\hline & град. $^{\circ}$ & рад & & & & & & \\
\hline 1 & 2 & 3 & 4 & 5 & 6 & 7 & 8 & 9 \\
\hline \multirow{9}{*}{$\mathrm{Be}(0)$} & 59,4 & 1,037 & $3081 \pm 5$ & $3415 \pm 10$ & $334 \pm 11$ & 0,108 & 0,0355 & 0,902 \\
\hline & 63,6 & 1,110 & $3081 \pm 5$ & $3395 \pm 10$ & $314 \pm 11$ & 0,10191 & 0,065 & 0,908 \\
\hline & 67,7 & 1,182 & $3081 \pm 5$ & $3347 \pm 10$ & $266 \pm 11$ & 0,08634 & 0,1034 & 0,9205 \\
\hline & 69,6 & 1,215 & $3081 \pm 5$ & $3338 \pm 10$ & $257 \pm 11$ & 0,0834 & 0,1136 & 0,923 \\
\hline & 76,3 & 1,332 & $3081 \pm 5$ & $3305 \pm 10$ & $224 \pm 11$ & 0,0727 & 0,145 & 0,9322 \\
\hline & 80,2 & 1,400 & $3081 \pm 5$ & $3317 \pm 10$ & $236 \pm 11$ & 0,0766 & 0,1481 & 0,9289 \\
\hline & 83,0 & 1,449 & $3146 \pm 10$ & $3392 \pm 10$ & $246 \pm 15$ & 0,0782 & 0,1515 & 0,9275 \\
\hline & 86,0 & 1,501 & $3081 \pm 5$ & $3362 \pm 10$ & $281 \pm 11$ & 0,0912 & 0,1431 & 0,9164 \\
\hline & 90,0 & 1,571 & $2992 \pm 5$ & $3297 \pm 10$ & $305 \pm 11$ & 0,1019 & 0,1398 & 0,9075 \\
\hline $\mathrm{BeO}(\times)$ & 90,0 & 1,571 & $3524 \pm 10$ & $3917 \pm 15$ & $393 \pm 18$ & 0,1115 & 0,1395 & 0,8997 \\
\hline
\end{tabular}

*) Для выбранного угла $\theta$ погрешность соответствующих ему чисел в столбцах 7-9 определяется наибольшей алгебраической суммой погрешностей столбцов 4, 6 .

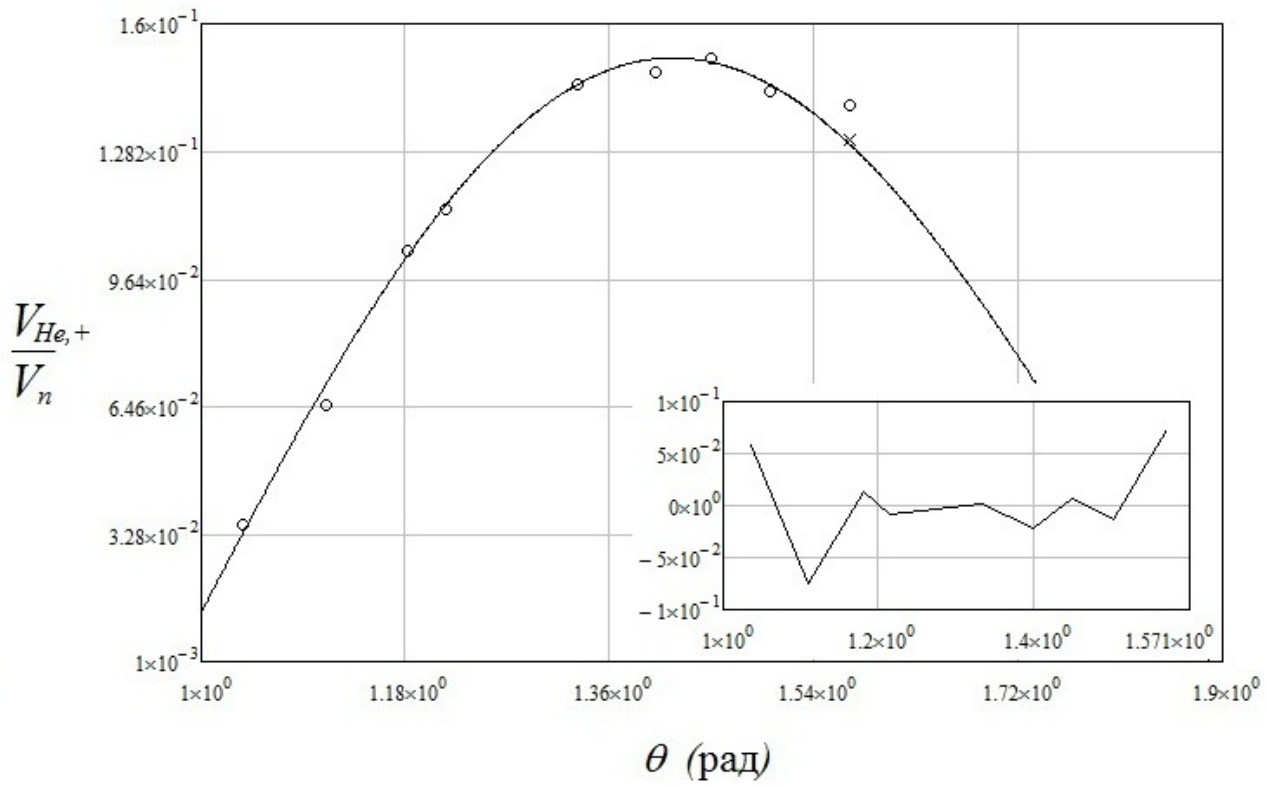

Рис. 5. Зависимость горизонтальной скорости атома гелия $V_{H e,+} / V_{n}$ в конвективной ячейке от угла наблюдения $\theta$ (рад).

Из представленных в таблице 2 экспериментальных результатов следует, что в ячейке скорость рассеивающего атома гелия зависит от угла наблюдения $\theta$. При малых углах наблюдения - скорость мала. С приближение угла наблюдения к определенному значению, в нашем случае около 83 , скорость принимает максимальное значение, а затем снова уменьшается.

Отмеченное выше изменение скорости атома гелия в зависимости от угла наблюдения можно объяснить, основываясь на зависимости горизонтальной скорости гелия II на верхней границе конвективной ячейки от $r$ : $\mathrm{v}_{r}\left(r, L_{z}\right) \propto J_{1}\left(\sigma_{1,1} r / L_{r}\right)(19)$, где $r$ - расстояния от центра ячейки до ее внешней границы. Такое объяснение возможно, если найти зависимость радиуса $r$ от угла наблюдения $\theta$.

Для нахождения этой зависимости запишем выражение для спирали Архимеда:

$$
\frac{\rho}{\rho_{m}}=\frac{\varphi}{\varphi_{m}}
$$

где $\rho$ - полярный радиус, $\varphi$ - полярный угол, $\rho_{m}=\sqrt{\left(L_{r} \sigma_{m} / \sigma_{1,1}\right)^{2}+L_{z}^{2}}=4,2313$ 
$\varphi_{m}=\operatorname{arctg}\left(L_{r} \sigma_{m} / \sigma_{1,1} / L_{z}\right)=0,6905$.

Вид спирали Архимеда (30) определяется геометрическими размерами конвективной ячейки.

Вблизи верхней границы ячейки, в точке с координатами $\rho_{m}, \varphi_{m}$ (например, точка $b$ на рис. 2), горизонтальная скорость атома гелия максимальна. При столкновении в этой точке нейтрона с движущимся атомом гелия, скорость рассеянного нейтрона $\left(V_{n 1} / V_{n}\right)_{1+}$ является наибольшей из (27), (28). Поэтому далее все расчеты по угловому распределению рассеянного нейтрона будем проводить для вылетающего из точки $\rho_{m}, \varphi_{m}$ быстрого нейтрона, т.к. только он фиксируется нейтронным спектрометром.

Перейдем в (30) к новым переменным. Полагаем $\rho=r+\rho_{m}, \varphi=\theta-\theta_{0}+\varphi_{m}$, что соответствует переносу точки $\rho_{m}, \varphi_{m}$ в полюс полярных координат, сдвинутый по углу $\theta$ по часовой стрелке на постоянный угол $\theta_{0}$. В этом случае радиус $r$ можно представить в виде:

$$
r=\frac{\rho_{m}}{\varphi_{m}}\left(\theta-\theta_{0}\right)
$$

где величина угла $\theta_{0}$ определяется из экспериментальных данных.

Подстановка (31) в (19) вблизи верхней границы ячейки приводит к выражению:

$$
\mathrm{v}_{r}\left(r, L_{z}\right)=V_{H e,+}=-A \frac{\pi}{L_{z}} \frac{L_{r}}{\sigma_{1,1}} J_{1}\left(\frac{\sigma_{1,1}}{L_{r}} \frac{\rho_{m}}{\varphi_{m}}\left(\theta-\theta_{0}\right)\right)=-A \frac{\pi}{L_{z}} \frac{L_{r}}{\sigma_{1,1}} J_{1}\left(\frac{3,83}{6,51} \frac{4,233}{0,691}\left(\theta-\theta_{0}\right)\right) .
$$

Таким образом, относительная горизонтальная скорость $V_{H e,+} / V_{n}$ будет определяться равенством:

$$
\frac{V_{H e,+}}{V_{n}}=-A^{\prime} J_{1}\left(4,1817\left(\theta-\theta_{0}\right)\right)
$$

где $A^{\prime}=\frac{A}{V_{n}} \frac{\pi}{L_{z}} \frac{L_{r}}{\sigma_{1,1}}$.

Подставляя экспериментальные данные таблицы 2 в выражение (33), и проводя оптимизацию теоретических расчетов и экспериментальных данных методом наименьшего среднеквадратичного отклонения, определим значения констант: $A^{\prime}=0,2603, \theta_{0}=0,9755$. Среднеквадратичное отклонение при этом достаточно мало, и составляет величину порядка $3,919 \cdot 10^{-3}$.

На рис. 5 сплошной линией показана оптимизированная кривая зависимости относительной горизонтальной скорости атома гелия $V_{H e,+} / V_{n}$ от угла наблюдения $\theta$. На вставке показано отклонение теоретической кривой от экспериментальных точек. Расчеты показывают, что величина отклонения не превышает 7,5\%.

Таким образом, в настоящем разделе показано, что рассеяние нейтрона на ротоне можно представить, как упругое столкновение нейтрона с атомом гелия, движущимся в цилиндрической конвективной ячейке. На это указывает количественное соответствие полученных ранее другими авторами экспериментальных данных по рассеянию нейтронов на гелии II с теоретическими расчетами рассеяния нейтрона на движущемся в конвективной ячейке атоме гелия.

\section{КОМБИНАЦИОННОЕ РАССЕЯНИЕ СВЕТА НА ГЕЛИИ II}

Наряду с описанным выше нейтронографическим анализом возможно исследование физических свойств ротонов с помощью комбинационного рассеяния света. В работе [22] приведены данные по измерению на дифракционном монохроматоре спектра, интенсивности и поляризации света методом комбинационного Рамановского рассеяния излучения аргонового лазера (длина волны 514,5 нм, мощность 1 Вт) сверхтекучим гелием при температурах в интервале $1,16^{\circ} \mathrm{K}$ и $2,14^{\circ} \mathrm{K}$.

В экспериментах падающий линейно поляризованный лазерный луч и рассеянный свет расположены в горизонтальной плоскости. Рассеянный свет собирался под углом $90^{\circ}$ к падающему излучению в пределах телесного угла около $0,08 \mathrm{cp}$. Для обеспечения максимального уровня обнаруженного сигнала вектор электрического поля падающего лазерного излучения ориентировался в горизонтальном направлении [23]. Анализ интенсивности спектра рассеянного излучения отчетливо показал наличие асимметричного резкого пика, сдвинутого на энергию $18.5 \pm 0.5^{\circ} \mathrm{K}$ относительно энергии падающего излучения.

В следующей работе по исследованию Рамановского рассеяния лазерного излучения сверхтекучим гелием [24] использовалась такая же экспериментальная установка, что и в первоначальной работе [22]. Однако, вместо дифракционного монохроматора использован спектрометр Фабри-Перо, чей свободный спектральный диапазон составлял величину $48,6 \mathrm{~K}$, что примерно соответствует тройному сдвигу двух - ротонного комбинационного рассеянного света. В качестве источника света использовался аргоновый ионный лазер (длина волны 488,0 нм). В этой работе показано, что сдвиг энергии рассеянного излучения относительно 
энергии падающего излучения равен $17,022 \pm 0,027 \mathrm{~K}$ при температуре $1,2{ }^{\circ} \mathrm{K}$.

Проведенные в работах [1-3] измерения показали, что исследование рассеяния света является более точным, по сравнению нейтронографическим, инструментом для описания элементарных возбуждений в сверхтекучем гелии. Очень высокое разрешение этого метода позволяет с достаточно точно измерять такие характеристики возбуждений в жидком гелии, как энергия и времена жизни ротона.

Приведенные выше результаты экспериментов по комбинационному рассеянию света опишем, исходя из предложенной в настоящей работе конвективной модели структуры ротона.

\section{Дипольный момент ротона}

Для определения дипольного момента ротона необходимо вычислить дипольный момент ускоренного атома, используя выражение [25]:

$$
\vec{d}(r, z)=\gamma \dot{\overrightarrow{\mathrm{v}}}(r, z)
$$

где $\dot{\overrightarrow{\mathrm{v}}}(r, z)$ - ускорение атома гелия, $\gamma=\frac{m_{H e} \alpha_{0}}{2 Z|e|}, \alpha_{0}=0,21 \cdot 10^{-24}$ см$^{-3}$ - поляризуемость атома гелия [26], $m_{\text {не }}$ и $Z=2$ - масса и заряд ядра атома гелия, $e$ - элементарный заряд. В (34) параметр $\gamma$ имеет величину $\gamma \approx 7,269 \cdot 10^{-40} \Gamma \cdot \mathrm{cm}^{3} /$ ед. зар. СГСЕ.

Из (34) и (19) определим инерционный дипольный момент движущегося по замкнутой траектории атома гелия. Исходя из аксиальной симметрии внутреннего строения ротона, его дипольный момент должен быть ориентирован в вертикальном направлении. Покажем это.

Будем считать, что в проходящей через ось ячейки плоскости движутся по замкнутым и симметричным относительно оси ячейки траекториям два атома гелия. Определим проекции их суммарных дипольных моментов:

$$
\begin{aligned}
& d_{r s}(r, z)=d_{r}(r, z)+d_{r}(-r, z)=\gamma\left[\dot{\mathrm{v}}_{r}(r, z)+\dot{\mathrm{v}}_{r}(-r, z)\right], \\
& d_{z s}(r, z)=d_{z}(r, z)+d_{z}(-r, z)=\gamma\left[\dot{\mathrm{v}}_{z}(r, z)+\dot{\mathrm{v}}_{z}(-r, z)\right],
\end{aligned}
$$

где $\dot{\mathrm{v}}_{r}(r, z)=\mathrm{v}_{r}(r, z) \frac{\partial \mathrm{v}_{r}(r, z)}{\partial r}+\mathrm{v}_{z}(r, z) \frac{\partial \mathrm{v}_{r}(r, z)}{\partial z}, \dot{\mathrm{v}}_{z}(r, z)=\mathrm{v}_{r}(r, z) \frac{\partial \mathrm{v}_{z}(r, z)}{\partial r}+\mathrm{v}_{z}(r, z) \frac{\partial \mathrm{v}_{z}(r, z)}{\partial z}$.

Учитывая зависимость проекций скорости $\mathrm{V}_{r}(r, z)$ и $\mathrm{V}_{z}(r, z)$ от координат $r$ и $z$ (19), нетрудно показать, что горизонтальный дипольный момент ротона равен нулю: $d_{r s}(r, z)=0$, а вертикальный - отличен от нуля $d_{z s}(r, z) \neq 0$.

Исходя из (35) можно утверждать, что движущиеся по замкнутым и симметричным относительно оси ячейки траекториям в одной плоскости два атома гелия находятся в связанном состоянии.

Суммарный дипольный момент двух связанных атомов гелия равен:

$$
d_{z s}(r, z)=\pi \frac{\gamma A^{2}}{L_{z}}\left[J_{0}^{2}\left(\frac{\sigma_{1,1} r}{L_{r}}\right)+J_{1}^{2}\left(\frac{\sigma_{1,1} r}{L_{r}}\right)\right] \sin \left(2 \pi \frac{z}{L_{z}}\right),
$$

и представляет собой два разнонаправленных диполя с цилиндрически симметричным распределением дипольного момента в пространстве.

Для оценки величины суммарного дипольного момента из (33) определим амплитуду скорости $A$ : $A=179,71 \mathrm{~m} / \mathrm{c}$.

Тогда максимальное значение суммарного дипольного момента для связанных атомов гелия достигается на оси ротона, и имеет порядок:

$$
d_{1}=\left|d_{z s}\left(0, \frac{L_{z}}{4}\right)\right|=0,226 \cdot 10^{-4} \mathrm{D} .
$$

Определим количество атомов гелия, которые участвуют в конвективном движении в ротоне.

Из расчетов следует, что в объеме одного ротона находится 7 атомов гелия:

$$
N_{\text {Rot }}=\frac{\rho}{m_{H e}} V_{R o t}=\frac{0,145 \frac{2}{\mathrm{CM}^{3}}}{6,646 \cdot 10^{-24} \mathrm{c}} 3,14 \cdot(5,61)^{2} \cdot 3,262 \cdot 10^{-24}=7,095,
$$

где $\rho=0,145 \Gamma / \mathrm{cm}^{3}[20]$ - плотность гелия II, $V_{R o t}=\pi L_{r}^{2} L_{z}$ - объем ротона.

Отсюда можно предположить, что в конвективном движении в ротоне участвует три пары связанных атома гелия (36), которые находятся в трех плоскостях, сдвинутых по азимуту на $120^{\circ}$.

Исходя из вышеизложенного, оценим величину максимального суммарного дипольного момента ротона в вертикальном направлении $D_{\max }=3 d_{1}=0,678 \cdot 10^{-4} \mathrm{D}$. В горизонтальном направлении, без учета седьмого атома 
гелия, дипольный момент ротона равен нулю.

Таким образом, приведенные выше оценки показывают, что дипольный момент ротона состоит из двух диполей, которые ориентированы в вертикальном направлении и направлены в противоположные стороны. Величина дипольного момента на оси ротона по порядку величины соответствует экспериментально измеренной [27].

\section{Седьмой атом гелия}

Из условия постоянства плотности гелия II следует, что седьмой атом гелия находится внутри ячейки и движется с тепловой скоростью, не покидая ее пределов. Для длительного удержания седьмого атома гелия в ротоне, и, учитывая симметрию задачи, необходимо потребовать, чтобы он двигался по круговой орбите относительно оси ячейки в плоскости $z=L_{z} / 2$. В этом случае наличие седьмого осциллирующего внутри ротона атома гелия приведет к тому, что общий горизонтальный дипольный момент ротона $d_{\Sigma}=d_{r s}+d_{r 7}$ будет отличным от нуля:

$$
d_{\Sigma}=d_{r s}+d_{r 7}=\gamma \dot{\mathrm{v}}_{r 7} .
$$

В выражении (39) ускорение атома гелия определяется выражением $\dot{\mathrm{V}}_{r 7}=-r \omega_{0}^{2} \cos \left(\omega_{0} t\right) \sin \left(\pi z / L_{z}\right)$, и задается зависимостью радиуса осциллятора от времени вида: $R_{o s}=r \cos \left(\omega_{0} t\right) \sin \left(\pi z / L_{z}\right)$, где $0 \leq r \leq 5,61 \AA$ радиус движения атома гелия внутри ротона, $\omega_{0}$ - частота осцилляций, $t$ - время.

Сделанное выше предположение о движении атома гелия по круговой орбите может быть физически обосновано действием Ван-дер-ваальсовых ориентационных сил [28], которые характеризуют взаимодействие между электрическими диполями ротона и седьмого атома гелия.

Полная энергия ориентационного взаимодействия диполей определяется суммой энергии взаимодействия диполя атома гелия с двумя разнонаправленными диполями ротона [28]:

$$
U(r)=-2 \gamma R^{-3}\left|d_{z r}\right|\left|d_{r 7}\right| \cos \left(\vartheta_{d}\right),
$$

где $R$ - расстояние между центрами диполей, $\vartheta_{d}$ - угол между направлениями диполей ротона и диполем атома гелия. Координаты центров диполей задаются в виде: для седьмого атома гелия $-\left(r, L_{z} / 2\right)$; для двух диполей ротона - $\left(0,(2 \pm 1) L_{z} / 4\right)$.

Исходя из выдвинутого выше предположения о длительном удержании седьмого атома гелия внутри ротона, можно утверждать, что расстояние между центрами диполей постоянно. Поэтому, полагая $R=R_{0}=$ const $_{1}$ и $\vartheta_{d}=\vartheta_{d 0}=$ const $_{2}$, определим из (40) координату минимума энергии ориентационного взаимодействия диполей.

На рис. 6 показана зависимость энергии ориентационного взаимодействия диполей ротона и атома в относительных единицах от расстояния $r / L_{r}$ от оси ротона.

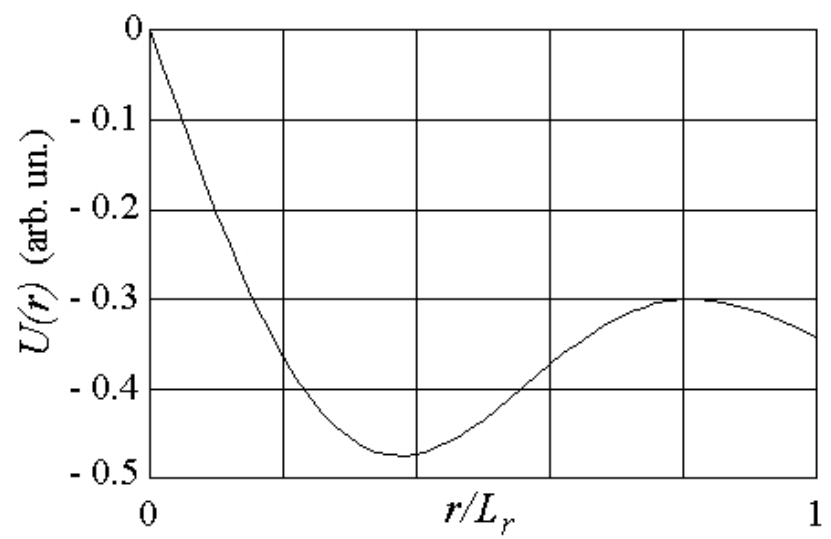

Рис. 6. Зависимость энергии ориентационного взаимодействия диполей ротона и атома гелия в относительных единицах от расстояния от оси ротона.

Из рисунка следует, что минимум энергии ориентационного взаимодействия диполей наблюдается на расстоянии $r=0,38 L_{r}$.

Отсюда следует оценка радиуса круговой орбиты седьмого атома гелия: $r=2,13 \AA$.

\section{Комбинационное рассеяние света на диполях гелия II}

Рассмотрим феноменологическую модель комбинационного рассеяния света на дипольных моментах ротонов в гелии II. Согласно предложенной выше модели строения ротона, ускоренные внутри него атомы 
гелия поляризованы под действием инерционных сил, которым можно привести в соответствие напряженность эффективного электрического поля $\vec{E}(r, z)$. Вектор электрической индукции в среде определяется через напряженность электрического поля и поляризацию среды: $\vec{D}(r, z)=\vec{E}(r, z)+4 \pi \vec{P}(r, z)$. В отсутствие внешних зарядов и токов, когда $\operatorname{div}(\vec{D})=0$ и $\frac{\partial \vec{D}}{\partial t}=0$, вектор электрической индукции равен нулю. Тогда напряженность электрического поля в ротоне определяется выражением:

$$
\vec{E}(r, z)=-4 \pi \vec{P}(r, z),
$$

где поляризация среды определяется выражением $\vec{P}(r, z)=3 d_{z s}(r, z) \vec{e}_{z}+d_{r 7} \vec{e}_{r}$, a $\vec{e}_{z}, \vec{e}_{r}-$ единичные вектора вдоль оси и радиуса ротона соответственно.

В рассеянии поляризованного лазерного света с ориентированным в направлении наблюдения вектором напряженности электрического поля принимает участие только горизонтальная составляющая поляризации среды, т.е. $P_{x}\left(0, L_{z} / 2\right)=d_{r 7}=-\gamma r \omega_{0}^{2} \cos \left(\omega_{0} t\right) \sin \left(\pi z / L_{z}\right)$.

Представим временную часть падающей волны лазерного света частоты $\Omega$ в виде $E(t)=E_{0} \cos (\Omega t)$. В поле этого излучения поляризуемость, а значит и параметр $\gamma$, можно представить в виде разложения по амплитудам малых возмущений [29]:

$$
\gamma(q) \simeq \gamma(0)+\left.\frac{d \gamma(q)}{d q}\right|_{q=0} q
$$

где $q=E_{0} \cos (\Omega t)$.

Тогда напряженность электрического поля рассеянного лазерного излучения принимает вид:

$$
E_{x}\left(\frac{L_{z}}{2}, q\right)=4 \pi r \omega_{0}^{2} E_{0}\left(\gamma(0) \cos \left(\omega_{0} t\right)+\gamma_{1}\left(\frac{L_{z}}{2}, 0\right)\left(\cos \left(\left(\Omega-\omega_{0}\right) t\right)+\cos \left(\left(\Omega+\omega_{0}\right) t\right)\right)\right),
$$

где $\gamma_{1}\left(\frac{L_{z}}{2}, 0\right)=\left.\frac{1}{2} \frac{d \gamma(q)}{d q}\right|_{q=0}$.

Как видно, в напряженности электрического поля рассеянного света (43) присутствует излучение с частотой, сдвинутой на $\omega_{0}$ от основной частоты $\Omega$. Исходя из (39) и экспериментальных данных [3], определим радиус движения седьмого атома гелия, при котором наблюдается сдвиг частоты на величину $\omega_{0}=17 K=35,4229 \cdot 10^{10} \quad$ Гц: $r \omega_{0}=\sqrt{\frac{2 k T}{m_{H e}}}=\sqrt{\frac{2 \cdot 1,38 \cdot 10^{-16} \frac{\text { эp }}{K} \cdot 1,2 K}{6,646 \cdot 10^{-24} 2}}=7059,4 \frac{c M}{c}$. Отсюда радиус движения седьмого атома гелия равен $r=R_{H e}=\frac{7059.4}{35,4229} 10^{-10} \mathrm{cM}=1,993 \mathrm{E}$. Полученная величина радиуса количественно соответствует той, которая приведена в предыдущем разделе: $r=2,13 \AA$.

Таким образом, рассеяние лазерного излучения на ротоне с описанными выше параметрами количественно соответствуют экспериментальным данным по комбинационному рассеянию света на гелии II. Такое соответствие указывает на возможность использования предложенной модели природы и структуры ротона для описания теплофизических свойств гелия II.

\section{ВЫВОДЫ}

В работе дан анализ существующих в настоящее время представлений о таких термодинамических характеристиках фононов и ротонов гелия II, как свободная энергия, энергия, количество, а также соответствующая им часть плотности нормальной компоненты гелия II. В настоящее время термодинамические параметры ротонов (свободная энергия, энергия, число ротонов, ротонная часть плотности нормальной компоненты гелия II) имеют неопределенное значение: либо энергия ротона в функции распределения Планка минимальна в окрестности некоторого значения импульса $p_{0} \neq 0$, либо она минимальна при нулевом импульсе $p_{0}=0$. Физически это означает то, что либо в гелии II равновесный энергетический спектр характеризуется направленным потоком ротонов, либо равновесный энергетический спектр ротонов беспотоковый.

В работе предложено рассматривать беспотоковый энергетический спектр для описания ротонной части нормальной компоненты гелия II, и дано физическое обоснование такого подхода. Это предложение основано на привлечении в описании физических процессов в гелии II такого процесса, как конвекция, который до настоящего времени в задачах о сверхтекучести не рассматривался. Оценочными расчетами показано, что 
термодинамические параметры гелия II (коэффициенты теплового расширения, кинематической вязкости и температуропроводности) соответствуют таким, при которых возможно формирование устойчивых цилиндрических конвективных ячеек типа ячеек Бенара.

Анализ процесса получения из жидкого гелия сверхтекучего гелия показывает, что при переходе через $\lambda$ точку конвективные процессы в переходном слое нормальной компоненты гелия II формируют горизонтальные слои толщиной порядка $h=3,262 \AA$, в которых плотно упакованы прямоугольные гексагональные призмы с такой же высотой и длиной ребра $c=3,243 \AA$. В такие гексагональные призмы вписаны цилиндрические конвективные ячейки высотой $h=L_{z}=3,262 \AA$ и диаметром $D_{r}=5,61 \AA$, которые представляют собой ротоны. Для таких конвективных ячеек описано пространственное распределение конвективной скорости атомов гелия. Определены вертикальные и горизонтальные пространственные периоды распределения ротонного газа в объеме гелия II. Показано, что из-за пространственной периодичности распределения ротонов, их энергетический спектр квантуется по вертикальному и горизонтальному импульсу (волновому числу). Такое квантование спектра позволяет с достаточной степенью точности описать экспериментальную зависимость энергетического спектра квазичастиц нормальной компоненты гелия II. Показано, что теоретически рассчитанная кривая роэнкро при беспотоковом ( $\left.p_{0}=0\right)$ энергетическом спектре в широком интервале температур (от 0,15 К до 1,95 К) наиболее точно соответствует экспериментальным данным. Для беспотокового энергетического спектра теоретически показано и экспериментально подтверждено, что рассеяние медленных нейтронов происходит не на ротонах, а на движущихся в них атомах гелия. Использование конвективной модели для описания рассеяния света на гелии II позволило определить дипольный момент ротона, а также количество участвующих в формировании ротона атомов гелия. Показано, что значение рассчитанного дипольного момента ротона по порядку величины совпадает с экспериментально измеренным. Оценочные расчеты показывают, что в формировании ротона в среднем участвуют семь атомов гелия. При этом показано, что два атома гелия в ротоне находятся в связанном состоянии, т.к. синхронно движутся с конвективной скоростью по замкнутым траекториям в проходящей через ось ротона вертикальной плоскости. Всего таких связанных пар - три, и они совершают движение в трех расположенных через равные углы по азимуту плоскостях. Согласно оценкам, седьмой атом гелия находится внутри ротона. Он движется в горизонтальной плоскости на полувысоте ротона по круговой траектории. Исходя из анализа энергии ориентационного взаимодействия диполей, дана теоретическая оценка. Полученная оценка радиуса движения седьмого атома гелия с достаточной степенью точности соответствует экспериментальным данным по рассеянию света на гелии II. В заключении сделан вывод о применимости конвективной модели природы и структуры ротона для описания физических свойств гелия II.

\section{СПИСОК ЛИТЕРАТУРЫ}

1. Landau L.D. Teoriya sverhtekuchesti geliya-II. Prilogenie [The theory of superfluidity of helium-II. Attachment] // UFN. 1967. - V. 93. - Iss. 11. - P. 495 - 520. (in Russian)

2. Cohen M., Feynman R.P. Theory of Inelastic Scattering of Cold Neutrons from Liquid Helium // Phys. Rev. - 1957. - Vol. 107. - No.1. - P.13-24.

3. Palevsky H., Otnes K., Larsson K.E. Excitation of Rotons in Helium II by Cold Neutrons // Phys. Rev. - 1958. - Vol. 112. No. 1. - P.11-18.

4. Yarnell J.L., Arnold G.P., Bendt P.J., Kerr E.C. Excitations in Liquid Helium: Neutron Scattering Measurements // Phys. Rev. 1959. - Vol.113. - No.6. - P.1379-1386.

5. Fizicheskaya encyklopedia [Physical encyclopedia] / Gl. red. A. M. Prohorov. Red.kol. D.M. Alekseev, A. M. Baldin, A. M. Bonch-Bruevich, A.S. Borovik-Romanov i dr. - M.: Sov. encyclopedia. T.I. Aaronova - Boma effekt - Dlinnie linii. - 1988. 704 p. (in Russian)

6. Feynman R.P. Atomic Theory of the Two-Fluid Model of Liquid Helium // Physical Review. - 1954. - Vol. 94. - No.2. P. 262-277.

7. Kruglov V.I., Collett M.J. Roton Excitation Spectrum in Liquid Helium II // Phys. Rev. Lett. - 2001. - Vol. 87. - No.18. P.185302.

8. Landau L.D. Teoriya sverhtekuchesti geliya-II [The theory of superfluidity of helium-II] // GETF. - 1941. - Vol. 11. P.592-616. (in Russian)

9. Halatnikov I.M. Teoriya sverhtekuchesti [The theory of superfluidity]. - M.: Nauka, 1971. - 320p. (in Russian)

10. Bozbiei L.S., Borts B.V., Kostikov A.O., Tkachenko V.I. Formation of Elementary Convective Cell in Horizontal Layer of Viscous Incompressible Fluid // East European J. of Phys.. - 2014. - Vol.1. - No.4. - P. 49-56.

11. Andronikashvili E.L. Issledovanie teplovoj strukturi geliya II s pomoschjyu rasseyaniya holodnih nejtronov [Study of the thermal structure of helium II using cold neutron scattering] // UFN. - 1960 - Vol. 72. - Iss. 4. - P. 697-710. (in Russian)

12. Gershuni G.Z., Zguhovitskij E.M. Konvektivnaya ustojchivost neszgimaemoj zgidkosti [Convective stability of an incompressible fluid]. - M: Nauka, 1972. - 393p. (in Russian)

13. Getling A.V. Formirovanie prostranstvennih struktur konvekcii Releya-Benara [Formation of Rayleigh-Benard's spatial convection structures] // UFN. -1991. - Vol.161. - Iss. 9. - P. 1-80. (in Russian)

14. Daunt Dzh.G, Smit P.S. Sovremennoe sostoyanie problemi zhidkogo geliya [The current state of the problem of liquid helium] // UFN. - 1955. - Vol. 56. - Iss. 3. - P. 249-416. (in Russian)

15. Kapitsa P.L. Sverhtekuchest geliya - II [Superfluidity of helium-II] // UFN. - 1967. - Vol. 93. - Iss. 11. - P. 481-494. (in 
Russian)

16. Meyer L., Mellink J.H. The Thermomechanical Effects in Liquid Helium II // Physica. - Vol. 13. - Iss. 4-5. - P. 197-215.

17. Svojstva elementov. Ch. I. Fizicheskie svojstva. Spravochnik [Properties of elements. Part I. Physical properties. Reference book]. Pod red. G.V. Samsonova. - M.: Metallurgiya, 1976. - 600p. (in Russian)

18. Polezgaev V.I., Soboleva E.B. Konvekciya Releya-Benara v okolokriticheskoj zgidkosti vblizi poroga ustojchivosti [RayleighBenard convection in a near-critical fluid near the stability threshold] // Izv. RAN. Mehanika zgidkosti i gaza. - 2005. - No. 2. P. 48-61. (in Russian)

19. Royal Society Mathematical Tables: Vol. 7. Bessel functions, P. Ш. Zeros and associated values. - Cambridge: Cambridge Univ. Press, I960. Russkij perevod: Tablici nulej Besselya. - M.: VC AN SSSR, 1967. - BMT; Iss. 44.

20. Donnelly R.J., Barenghi C.F. The Observed Properties of Liquid Helium at the Saturated Vapor Pressure // J. Phys. Chem. Ref. Data. - 1998. - Vol. 27. - No. 6. - P. 1217-1274.

21. Fizicheskaya encyklopedia [Physical encyclopedia] / Gl. red. A.M. Prohorov. Red.kol. D.M. Alekseev, A.M. Baldin, A.M. Bonch-Bruevich, A.S. Borovik-Romanov i dr. - M.: Bolschaya Rossijskaya encyclopedia. Vol. 3. Magnitoplazmennij Pojntinga teorema. - 1992. 672 p. (in Russian)

22. Greytak T.J., Yan J. Light Scattering From Rotons In Liquid Helium // Physical Review Letter. - 1969. - Vol. 22. - No. 19. P. $987-990$.

23. Greytak T.J. Light Scattering from Superfluid Helium. Quantum Liquids. Lectures presented at the International School of Low Temperature Physics. Edited by J. Ruvalds and T. Regge. Erice, Italy, June 11-25, 1977.

24. Greytak T. J., Woerner R., Yan J., Benjamin R. Experimental Evidence for a Two-Roton Bound State in Superfluid Helium // Physical Review Letters. - 1970. - Vol. 25. - No. 22. - P.1547 - 1550.

25. Melnikovsky L.A. Polarization of Dielectrics by Acceleration // J. Low Temp. Phys. - 2007. - Vol. 148. - P. 559-564.

26. Spravochnik himika. Tom 1. Obschie svedeniya, stroenie veschestva, svojstva vazgnejshih veschestv, laboratrnaya tehnika [Reference book of the chemist. Volume 1. General information, the structure of matter, the properties of critical substances, laboratory equipment] / Pod red. B.P. Nikolskogo. - M.-L.: Himiya, 1966 - 1071 p. (in Russian)

27. Poluektov Yu.M., Savchenko V.N. On Electric Polarization of Helium Atoms by Acceleration / PAST, Series: Nuclear Physics Investigations (57) - 2012. - No. 1. - P. 299-301.

28. Smirnova N.A. Metodi statisticheskoj termodinamiki $\mathrm{v}$ fizicheskoj himii [Methods of statistical thermodynamics in physical chemistry]. - M.: Vish. Shkola, 1982. - 455p. (in Russian)

29. Fabelinskij I.L. Otkritie kombinatcionnogo rasseyaniya sveta [The discovery of Raman scattering of light] // UFN. - 1978. Vol. 126. - Iss. 1. - P. 124 - 152. (in Russian) 